\title{
Aerosol Properties of the Atmospheres of Extrasolar Giant Planets
}

\author{
P. Lavvas ${ }^{1}$ and T. Koskinen ${ }^{2}$ \\ ${ }^{1}$ Groupe de Spectrométrie Moléculaire et Atmosphérique, UMR CNRS 7331, Université de Reims \\ Champagne Ardenne, Reims, France; panayotis.lavvas@univ-reims.fr \\ ${ }^{2}$ Lunar and Planetary Laboratory, University of Arizona, Tucson, AZ, USA \\ Received 2017 June 27; revised 2017 August 16; accepted 2017 August 25; published 2017 September 20
}

\begin{abstract}
We use a model of aerosol microphysics to investigate the impact of high-altitude photochemical aerosols on the transmission spectra and atmospheric properties of close-in exoplanets, such as HD 209458 b and HD 189733 b. The results depend strongly on the temperature profiles in the middle and upper atmospheres, which are poorly understood. Nevertheless, our model of HD 189733 b, based on the most recently inferred temperature profiles, produces an aerosol distribution that matches the observed transmission spectrum. We argue that the hotter temperature of HD $209458 \mathrm{~b}$ inhibits the production of high-altitude aerosols and leads to the appearance of a clearer atmosphere than on HD 189733 b. The aerosol distribution also depends on the particle composition, photochemical production, and atmospheric mixing. Due to degeneracies among these inputs, current data cannot constrain the aerosol properties in detail. Instead, our work highlights the role of different factors in controlling the aerosol distribution that will prove useful in understanding different observations, including those from future missions. For the atmospheric mixing efficiency suggested by general circulation models, we find that the aerosol particles are small $(\sim \mathrm{nm})$ and probably spherical. We further conclude that a composition based on complex hydrocarbons (soots) is the most likely candidate to survive the high temperatures in hot-Jupiter atmospheres. Such particles would have a significant impact on the energy balance of HD 189733 b's atmosphere and should be incorporated in future studies of atmospheric structure. We also evaluate the contribution of external sources to photochemical aerosol formation and find that their spectral signature is not consistent with observations.
\end{abstract}

Key words: meteorites, meteors, meteoroids - planets and satellites: atmospheres - planets and satellites: composition - planets and satellites: gaseous planets - planets and satellites: individual (HD 209458 b, HD 189733 b)

\section{Introduction}

The study of exoplanetary atmospheres is rapidly expanding, providing new insights into the complexity and diversity of the atmospheres of these distant planets. Apart from the chemical inventories observed on these objects, recent studies suggest that subsequent products of chemical evolution such as hazes can also exist in these atmospheres. Hazes can strongly influence the thermal structure, dynamics, and photochemistry, as observations of solar system atmospheres have demonstrated (West et al. 2009, 2013). Depending on their formation mechanism, hazes can be separated into being of either photochemical or condensate nature. Here we investigate the possible properties of hazes of photochemical origin in the atmospheres of extrasolar giant planets (EGPs), based on available observations.

Among the most observed exoplanets are the hot Jupiters HD 209458 b and HD 189733 b. Primary eclipse observations of HD $189733 \mathrm{~b}$ at UV, visible, and IR wavelengths by the different instruments of the Hubble Space Telescope (HST) and Spitzer suggest a rather monotonic decrease in the transit depth with increasing wavelength (Pont et al. 2008; Sing et al. 2009, 2011, 2016). This wavelength dependence is contrary to the sharp molecular signatures anticipated at nearIR wavelengths of $\mathrm{H}_{2} \mathrm{O}, \mathrm{CH}_{4}, \mathrm{CO}$, and $\mathrm{CO}_{2}$, which appear in secondary eclipse observations of this atmosphere (Grillmair et al. 2008; Désert et al. 2009; Swain et al. 2009). Primary transit observations probe higher altitudes than the secondary eclipse; therefore, these observations were interpreted as indications for the presence of a silicate condensate (enstantite, $\mathrm{MgSiO}_{3}$ ) at the pressure levels probed (Lecavelier Des Etangs et al. 2008a). However, subsequent studies of cloud microphysics demonstrate that silicate clouds cannot easily reproduce the observed primary transit signature (Lee et al. 2015; Pinhas \& Madhusudhan 2017), and thus a different type of heterogeneous opacity is required.

On the other hand, the primary transit spectrum of $\mathrm{HD}$ $209458 \mathrm{~b}$ includes signatures of Rayleigh scattering, Na, and $\mathrm{H}_{2} \mathrm{O}$, and it is roughly consistent with a clear atmosphere model (Lavvas et al. 2014). HD 209458 b is known to have a very low visible albedo (Rowe et al. 2006), and this characteristic could be caused by absorption from $\mathrm{TiO}$ or $\mathrm{VO}$ (Hubeny et al. 2003; Désert et al. 2008; Lecavelier Des Etangs et al. 2008b), which are known to have large absorption crosssections at visible wavelengths and can be present in the deep troposphere. Similarly, any gaseous species absorbing in the visible with an absorption opacity larger than the scattering opacity by clouds would have the same effect (Marley et al. 1999; Sudarsky et al. 2000). Theoretical temperature profiles including heating by such molecules (Showman et al. 2009) are consistent with the secondary eclipse observations (Diamond-Lowe et al. 2014; Line et al. 2016). Other possible visible extinction candidates include sulfur photochemical products (Zahnle et al. 2009) and silicate clouds (Fortney et al. 2003). Therefore, current observations and modeling suggest that a high-altitude heterogeneous opacity similar to that on HD $189733 \mathrm{~b}$ is not required to explain the observations of HD 209458 b.

It is clear that the analysis of these observations is difficult due to the presence of large systematic and random uncertainties for the different instruments involved. For 
example, the near-IR observations of HD $189733 \mathrm{~b}$ do suggest the presence of some gaseous absorbers, but the uncertainties of the analysis do not provide a clear picture (Swain et al. 2008; Gibson et al. 2011). Therefore, a definite interpretation of the available observations might prove equivocal, and repeated observations are required to resolve such issues. Notwithstanding these obstacles, we can investigate the properties of hazes in hot-Jupiter atmospheres and potentially aid in the interpretation of the observations.

Given the limited information we have for hazes in exoplanet atmospheres, we can first consider what we know about hazes from the atmospheres of the solar system. Numerous studies have demonstrated the multiple ways in which hazes can affect atmospheric evolution and planetary surface properties, from short- to long-term aspects. The most characteristic example is our own planet (through climate change), as well as Saturn's moon Titan, where hazes are the dominant feature of the atmosphere and are known to affect the thermal structure, photochemistry, and surface properties (see the recent review by West et al. 2013). The most relevant examples, however, are the cases of the giant planets, which are also affected by different kinds of hazes.

On Uranus and Neptune, scattering by small haze particles affects the albedo and the thermal structure of their atmospheres (Marley \& McKay 1999). On Jupiter and Saturn, for which more observations are available, hazes can be separated into two general families: photochemical aerosols and clouds (West et al. 2007, 2009). Photochemical aerosols are present in the stratosphere and upper troposphere of both giant planets, while clouds are formed at deeper regions. The composition of photochemical aerosols has not been clearly identified yet. Both observations and modeling suggest that they are hydrocarbon based (Ben-Jaffel et al. 1995; Wong et al. 2003; Koskinen et al. 2016), but contributions from phosphine chemistry on Saturn and sulfur chemistry on Jupiter are also anticipated, although not yet verified. Average particle sizes range between $\sim 0.01 \mu \mathrm{m}$ in the stratosphere to $\sim 0.1 \mu \mathrm{m}$ in the upper troposphere. At deeper layers, the condensation of ammonia, ammonia sulfide, and water dominate the particulate extinction in these atmospheres, and photochemical aerosols produced at higher altitudes act as nucleation sites for the production of cloud particles that eventually grow to sizes of a few microns. A similar stratification can be anticipated for hazes in exoplanet atmospheres, although the chemical composition of the aerosols and the gaseous species condensing into clouds could be different (Marley et al. 2013).

Given our current understanding of the role of hazes in planetary atmospheres, and motivated by the above observations of HD $189733 \mathrm{~b}$, we would like to understand how hazes would behave in exoplanet atmospheres. In principle, such an investigation would require detailed studies addressing multiple aspects of haze properties, which can be specific to each planet. However, at the current early stage of this investigation, we can first attempt to address the basic questions common to all exoplanet cases, such as:

1. What kind of hazes forms in exoplanet atmospheres and under which conditions? Why do they seem to be present in one case (HD 189733 b) and absent in another (HD 209458 b)?

2. What are the processes that define the production and evolution of hazes in these environments, and how do these compare with what we already know from the atmospheres of our solar system?

3. If hazes do exist in exoplanet atmospheres, what would be their size, their density, and their optical properties? What methods are required to detect them in future observations?

4. How would the hazes interact with their atmospheric background? What would be their role in the thermal structure of the atmosphere and their interaction with atmospheric circulation, and how would they affect the atmospheric composition (e.g., heterogeneous chemistry, photochemistry)?

Definite answers to these question are constrained by the limited number of observations currently available, as well as the multitude of environmental conditions among various planets. Nevertheless, we can utilize the methods and knowledge we have gained from solar system studies, along with the current understanding of exoplanet atmospheric properties, in order to address some of the above questions.

We note that Helling \& Woitke (2006) also presented detailed models for the formation and evolution of clouds in brown dwarf atmospheres. These were subsequently applied to the atmospheric conditions in EGPs in order to investigate the composition and optical properties of clouds in these environments in relation to available observations (Lee et al. 2015), the impact of clouds on the atmospheric structure (Lee et al. 2017), and the possible occurrence of lightning in these cloud structures (Helling et al. 2013). Instead of the physics of cloud formation, in the current study we focus on the properties of the photochemical aerosols that can form in the upper atmosphere of EGPs.

We use a 1D aerosol microphysics model that we adapted to the environments of EGPs. We apply this model to the two representative cases of EGPs: HD 209458 b and HD 189733 b, for which we have a better understanding of their atmospheric properties relative to those of other planets. We note that our study focuses only on the photochemically produced aerosols and does not follow the interaction of these aerosols with the background gases, which can condense and lead to the formation of cloud particles. As outlined by the nature of the questions raised above, our goal in this study is not to provide a detailed analysis for each planet separately, but more interestingly, to investigate the phase space of the resulting aerosol properties in such atmospheres. This investigation provides a basic background for aerosol properties in exoplanet atmospheres, on which planet-specific investigations will further advance our knowledge.

\section{Model Description}

We use a 1D aerosol microphysics model to investigate the properties of aerosol particles in extrasolar atmospheres. Our prototype model is described in detail in Lavvas et al. (2010), where it was used for the interpretation of Titan's in situ aerosol observations obtained with the Huygens probe. Here, the model is updated to represent the atmospheric conditions found on EGPs. In a nutshell, the model utilizes a geometrically expanding grid of particle sizes and calculates the population of each particle size by solving the continuity 
equation

$$
\begin{aligned}
\frac{\partial n_{p}}{\partial t}= & -\frac{1}{r^{2}} \frac{\partial\left(r^{2} \Phi\left(v_{p}\right)\right)}{\partial r} \\
& +\frac{1}{2} \sum_{i=1}^{p-1} K_{\mathrm{B}}\left(u_{i}, v_{p}-u_{i}\right) n\left(u_{i}\right), n\left(v_{p}-u_{i}\right) \\
& -n_{p} \sum_{i=1}^{N_{\max }} K_{\mathrm{B}}\left(u_{i}, v_{k}\right) n\left(u_{i}\right)+\left.\frac{\partial n\left(v_{p}\right)}{\partial t}\right|_{P C},
\end{aligned}
$$

where $n\left(v_{p}\right)$ and $\Phi\left(v_{p}\right)$ are the number density and flux of volume $v_{p}$ particles, respectively, $r$ is the planetocentric radius, and $K_{\mathrm{B}}$ is the coagulation kernel that is dominantly controlled by the random collisions among particles (Brownian coagulation dominates over other processes for submicron-size particles, which as we will see is the case for the resulting aerosol particle sizes). The first sum on the right-hand side describes the production of particles of size $v_{p}$ from smaller particles, the second sum is the loss of particles of size $v_{p}$ by coagulation to form bigger particles, $\partial n\left(v_{p}\right) /\left.\partial t\right|_{P C}$ describes the local photochemical production of this size of particles (only for the first bin particles), and $N_{\max }$ is the number of volume bins considered in the calculations. We use 30 bins with an expanding bin structure covering the range between $1 \mathrm{~nm}$ and $\sim 1 \mu \mathrm{m}$ particle bulk radii (the radius of an equivalent-mass spherical particle if the assumed shape is not spherical). The particle vertical transport is controlled by their gravitational settling velocity and the influence of atmospheric mixing, which is described through an eddy diffusion profile. The combined effect on the flux, $\Phi\left(v_{p}\right)$, of the particles is

$$
\Phi\left(v_{p}\right)=-V_{s} n\left(v_{p}\right)-K_{\mathrm{ZZ}} n \frac{\partial\left(n\left(v_{p}\right) / n\right)}{\partial r}
$$

where $V_{s}$ is the settling velocity of the particles $p$ (see Lavvas et al. 2010), $K_{\mathrm{ZZ}}$ is the eddy mixing profile, and $n$ is the atmospheric density. Below we discuss in detail the specific parameters that affect the results of our calculations.

\subsection{Particle Collisions}

Before evaluating the possible aerosol particle growth in exoplanet atmospheres, we need to confirm that collisions among particles will permit their coagulation. Although this assumption is valid for solar system studies where the particle thermal velocities are small and collision energies are low, the high temperatures of EGPs and their resulting high thermal velocities could limit the sticking efficiency among particles. The critical factor is the bond energy between the colliding particles, which depends on the material they are made of. As the latter parameter is not known, we can evaluate the bond strength under different composition assumptions and compare it with the collision energies anticipated in exoplanet atmospheres.

For this task, we use the approach developed for collisions among dust particles in the interstellar medium (Chokshi \& Tielens 1993; Dominik \& Tielens 1997) that is based on the surface energy and contact theory of solids (Johnson et al. 1971). The approach utilizes macroscopic parameters for the elastic properties of the particle's material such as Young's modulus $E$, surface energy $\gamma$, and Poisson ratio $\nu$, and allows for the evaluation of the maximum collision velocity of

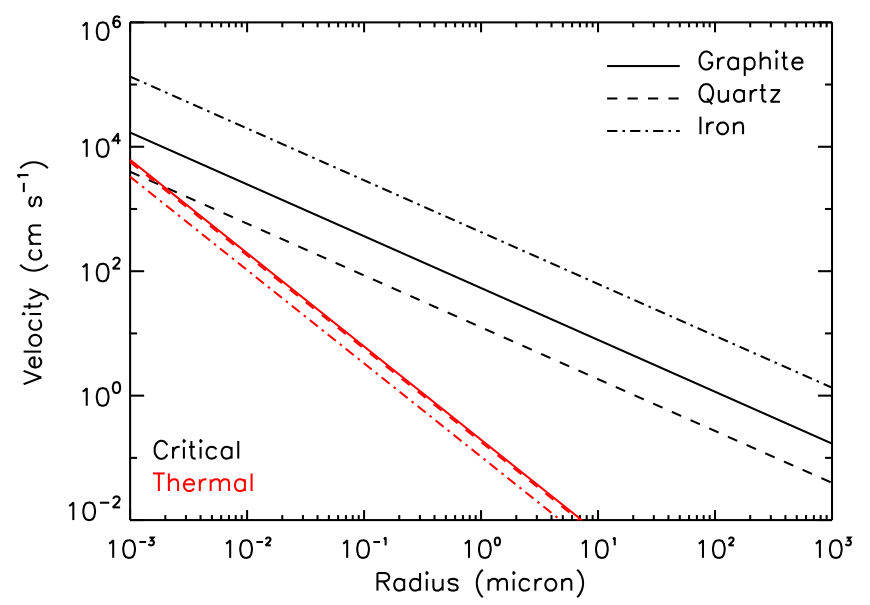

Figure 1. Variation of critical velocity (black) and thermal particle velocity (red) at $T=1000 \mathrm{~K}$ with particle radius, for different compositions. Parameters for the evaluation of the critical velocity for each composition are taken from Chokshi \& Tielens (1993).

two spherical particles for which the particles will stick together. The application of this approach to exoplanet conditions demonstrates that for an atmospheric temperature of $T=1000 \mathrm{~K}$, particle collisions would allow sticking and thus growth for compositions similar to quartz, iron, and graphite (Figure 1). These are characteristic examples of possible chemical compositions that could exist in EGP aerosols and provide an overall picture of the limits of particle collision energies that allow their microphysical growth. Only for very small particle radii, below $\sim 3 \mathrm{~nm}$, will the critical and thermal velocities for quartz composition approach each other, which would imply that particles would have to reach this limiting radius through interaction with the gas phase (condensation or chemistry) before coagulation can permit further growth. However, at such small sizes, the use of macroscopic theory is questionable, while other theoretical studies demonstrate that at this size range, long-range interactions, such as van der Waals forces, are important and can significantly enhance the coagulation rates (Harris \& Kennedy 1988). Temperature changes give rise to small changes in the above picture, thus we conclude that particle collisions in exoplanet atmospheres can result in their coagulation.

\subsection{Particle Ablation}

The model described above could be directly applied to the giant planets of our solar system since it encompasses the main processes controlling the aerosol particle evolution in the pressure regions probed by observations. However, for EGPs, we need to further consider the impact of the high temperatures observed in their atmospheres on the properties of the produced aerosol particles. For the gaseous species of the atmosphere, we know that high temperatures drive their thermal decomposition. A similar procedure can affect the aerosol particles when they reach atmospheric regions with significantly warmer temperatures than those at their formation region. We can treat this process as an ablation procedure in which case the particles lose mass at a rate $m_{p}$ that depends on the atmospheric temperature, the size of each particle, and the vapor pressure of their material. We included this mechanism in our calculations by adding one more term to the continuity equation described 


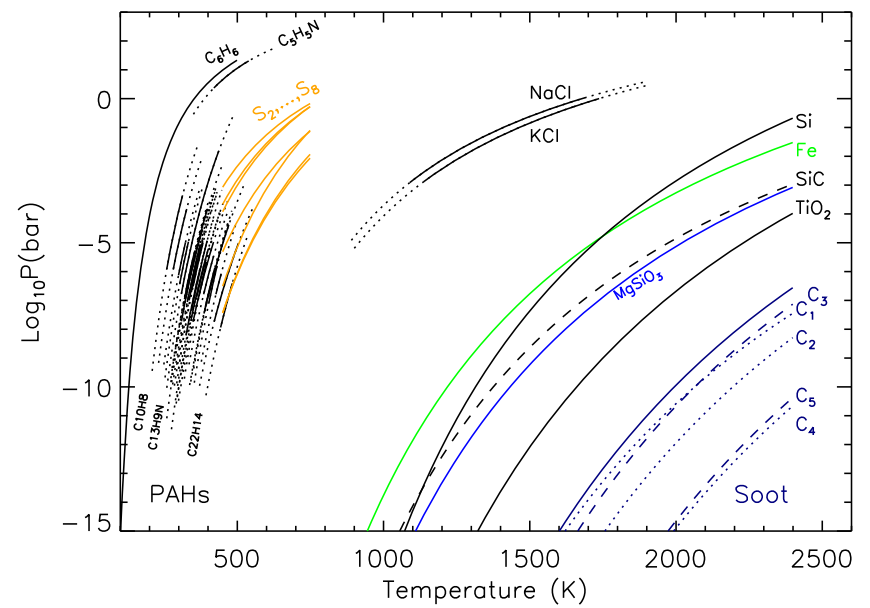

Figure 2. Saturation vapor pressure curves of different compounds. Parameters for aromatic compounds are taken from Oja \& Suuberg (1998) and Goldfarb \& Suuberg (2008), and for sulfur allotropes from Lyons (2008). The Si (black) and $\mathrm{Fe}$ (green) curves present the saturation vapor pressure over liquid $\mathrm{Si}$ (NIST) and solid Fe (Visscher et al. 2010), respectively. For enstantite (blue curve), we use the vapor pressure estimated in Cameron (1985). Parameters for $\mathrm{SiC}$ and $\mathrm{TiO}_{2}$ are taken from Lilov (1993) and Woitke \& Helling (2004), respectively. For soot (navy curves), saturation vapor pressures are from Michelsen et al. (2007), with the different broken lines representing the contribution of various carbon clusters. $\mathrm{NaCl}$ and $\mathrm{KCl}$ curves are from NIST.

previously:

$$
\left.\frac{\partial n_{p}}{\partial t}\right|_{\text {Ablation }}=-\frac{\partial\left(\dot{m}_{p} n_{p}\right)}{\partial m}
$$

This procedure is similar to the description of evaporating cloud particles and more details for the evaluation of all terms in the above equations can be found in Lavvas et al. (2011a).

The importance of aerosol ablation depends on the chemical composition of the particles, which controls their resistance at high temperatures. Figure 2 demonstrates this sensitivity by presenting the saturation vapor pressure curves of different compounds. Included are characteristic examples of polycyclic aromatic compounds, which are believed to contribute to the photochemical aerosols of Jupiter and Saturn (Wong et al. 2000, 2003) and the aerosols in Titan's atmosphere (Lavvas et al. 2011b), while they are also observed in the soots formed from combustion engines (Frenklach 2002), as well as in the interstellar medium dust (Draine 2004). These are mainly based on carbon (and nitrogen for Titan and the ISM) chemistry. However, the hot environments of exoplanets are potentially rich in more exotic species such as $\mathrm{S}, \mathrm{Si}, \mathrm{Ti}$, and other metals that could also lead to the production of complex molecules and eventually form aerosol particles. For example, silicon species such as silane $\left(\mathrm{SiH}_{4}\right)$ and its photochemical products have a similar chemical complexity to the more familiar (from the atmospheric chemistry point of view) hydrocarbons derived from the photolysis of methane, while sulfur compounds have already been proposed as possible components of exoplanetary aerosols (Zahnle et al. 2016).

Silicon carbide ( $\mathrm{SiC}$ ) is another candidate that is usually found in carbon- rich stars, while other silicate mixtures such as enstantite $\left(\mathrm{MgSiO}_{3}\right)$, forsterite $\left(\mathrm{Mg}_{2} \mathrm{SiO}_{4}\right)$, quartz $\left(\mathrm{SiO}_{2}\right)$, and oxides of titanium or vanadium $\left(\mathrm{TiO}_{2}, \mathrm{VO}\right)$ are usually considered as possible contributors to the formation of condensates in the deeper regions of exoplanet atmospheres (e.g., Lecavelier Des Etangs et al. 2008a), and we include them here for comparison. Recent studies on the physical properties of such condensates demonstrate that they cannot reproduce well the observed primary transit spectra for HD $189733 \mathrm{~b}$ (Lee et al. 2015; Pinhas \& Madhusudhan 2017), although they can satisfy secondary eclipse constraints (Lee et al. 2017). These results indicate that a heterogeneous compound with a different chemical composition relative to the dominant silicate condensates in the lower atmosphere is required in the upper atmosphere.

Among the compounds we investigated, the material with the highest resistance to extreme temperatures is soot. Although this term encompasses a large family of combustion or pyrolysis products of carbon chemistry, all characteristic examples we found have the lowest rates of evaporation at high temperatures. Soot is a common product of hightemperature chemistry, but its formation in a planetary atmosphere will depend on the availability of carbon species. A soot-composition haze has been suggested for the cooler atmosphere $\left(T_{\text {eff }} \sim 550 \mathrm{~K}\right)$ of GJ 1214 b (Morley et al. 2013). Here we assume a soot composition and explore the possibility of such a photochemical aerosol formation in hot exoplanets and the resulting particle properties.

\subsection{Aerosol Production}

In order to initiate the simulation of aerosol evolution, we need to assume an aerosol production profile that generates particles in the first bin of the aerosol size grid. If we assume that the production profile can be represented by a Gaussian distribution in pressure, then we need to specify the location of the peak, the width of the distribution, and the magnitude of aerosol production, i.e., the mass production rate of aerosols. Although we do not have any observational constraints for these properties, we can derive some indicative values based on photochemical models and what we know from the solar system. Taking as example the atmosphere of Jupiter, the mass flux of aerosols inferred from observations $(\sim 7 \times$ $10^{-14} \mathrm{~g} \mathrm{~cm}^{-2} \mathrm{~s}^{-1}$ ) corresponds to about $1 \%$ of the total mass flux generated by the photolysis of methane (Moreno 1996; Wong et al. 2000). The ion-neutral processes leading to the formation of photochemical aerosols in Titan's upper atmosphere have a similar efficiency, although the total mass flux of aerosols is further increased through neutral chemical processes and reaches about $10 \%$ of the mass flux generated by the photolysis of Titan's major atmospheric compounds, $\mathrm{N}_{2}$ and $\mathrm{CH}_{4}$ (Lavvas et al. 2013). Thus, we can start our simulations by assuming a similar range of efficiencies for the production of aerosols in exoplanets. The location of the aerosol production profile can also be estimated from the photochemical model results. These suggest that most of the high-energy photons are deposited in the upper atmosphere, above the $1 \mu \mathrm{bar}$ level. Hence, we center our aerosol production profile on this location and assume a profile width that ranges between 0.1 and $10 \mu$ bar.

\subsection{Particle Shape}

The shape of the particles depends on the phase of their material (liquid/solid), as well as their interaction with the gasphase background; the coagulation of liquid particles results in the formation of a larger spherical particle, while for solid particles collisions lead to their aggregation. However, in the latter case, heterogeneous processes at the surface of the 
particles (e.g., chemical reactions, adsorption, condensation) can modify aggregates toward a quasi-spherical shape, if the mass added heterogeneously from the gas phase is comparable to the mass added by coagulation (Mitchell \& Frenklach 2003; Morgan et al. 2007; Lavvas et al. 2011a, 2011b). Thus, the theoretical evaluation of the particle shape requires an in depthknowledge of the mechanism(s) generating the particles, which is lacking at the current level of aerosol investigation for exoplanet atmospheres. We perform our calculations assuming spherical particles, and we subsequently conclude that aggregate structures are not very likely in the upper atmosphere but may form in the lower atmosphere where conditions for aggregation are more favorable.

\subsection{Particle Charge}

Another parameter that affects the growth of particles is the potential charge on their surface. Collisions among charged particles can increase or decrease the resulting particle size, if the particles have different or the same sign charge, respectively. Typically, the effect of particle charging is taken into account through a charge density parameter, $\chi$, which describes the total number of charges, $Z$, on the surface of a particle as a function of its bulk radius, $r,(Z=\chi r)$. Subsequently, the total charge of the particles is used for the calculation of the particle sticking efficiency, $\alpha$ (e.g., Lavvas et al. 2010). Typical values for $\chi$ range between 10 and $30 \mathrm{e}^{-} \mu \mathrm{m}^{-1}$ in the solar system aerosols, which means that electrostatic effects start to modify the particle growth for sizes above $1 \mu \mathrm{m}$, for the typical temperatures in the solar system. For the higher temperatures found in EGPs, the corresponding effect of charging would be significantly reduced if $\chi$ had a similar magnitude. However, charging rates for extrasolar planets could be significantly different from those in our solar system due to the presence of high densities of free electrons from the photoionization of $\mathrm{Na}$ and $\mathrm{K}$ (Lavvas et al. 2014), and due to the possibly higher impact of the photoelectric effect on particle ionization, resulting from the enhanced stellar fluxes in EGP environments. In our calculations, we assume that the charging effects are negligible $(\chi=0)$, and we subsequently demonstrate that this assumption is valid for the upper atmosphere probed by the transit observations, due to the small size of the simulated particles that is controlled by other processes.

\section{Case Study}

We proceed now to the application of the model to HD 189733 b and HD 209458 b. We use the Lavvas et al. (2014) exoplanet chemistry kinetics model for the simulation of the atmospheric composition from which we derive the potential aerosol mass fluxes for each planet (see Vardavas \& Taylor 2007 and Lavvas et al. 2008 for more details on the chemistry model). The background chemistry depends strongly on the assumed elemental abundance of each system, the stellar insolation reaching the planetary atmosphere, the atmospheric thermal structure, and the efficiency of the atmospheric mixing. We consider solar elemental abundances in our calculations. For the stellar flux, we use a compilation based on the $\epsilon$-Eridani spectrum, which has a similar spectral type to HD 189733 b, and a Phoenix model of appropriate parameters for this star. For HD 209458, we use the solar spectrum (see Lavvas et al. 2014). Current temperature profiles suggested for HD

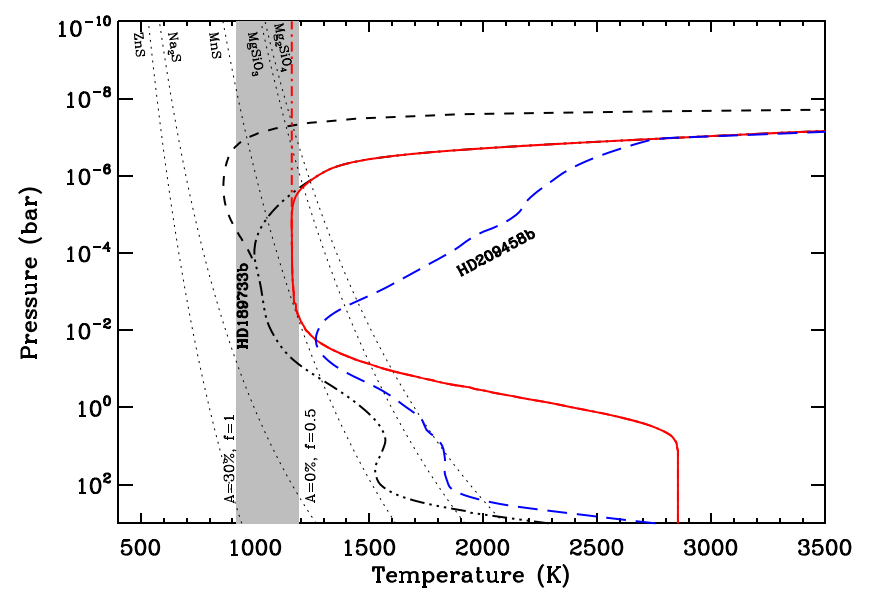

Figure 3. Temperature profiles for the atmospheres of HD 209458 b (blue, long-dashed line, MK case) and HD 189733 b (black dashed line, M profile; black dashed-triple-dotted line, MK profile; red solid line, LK profile; red dashed-dotted line, L profile; see the text for details) corresponding to day average illumination conditions. The gray band presents the range of skin temperatures anticipated for HD 189733 b, bound by a high Bond albedo case $(A=30 \%)$ with complete redistribution of incoming radiation $(f=1)$, and a low albedo case $(A=0 \%)$ with no redistribution to the night side $(f=1 / 2)$. Dotted lines show the conditions for the formation of condensates $\mathrm{ZnS}, \mathrm{Na}_{2} \mathrm{~S}$, $\mathrm{MnS}, \mathrm{MgSiO}_{3}$, and $\mathrm{Mg}_{2} \mathrm{SiO}_{4}$, assuming thermochemical equilibrium everywhere in the atmosphere and solar metallicity (Visscher et al. 2010).

$189733 \mathrm{~b}$ include the studies by Line et al. $(2010,2014)$ and Moses et al. (2011). Here we only consider the two limiting cases of the later two studies. The Moses et al. (2011) $p-T$ profile (corresponding to day averaged conditions and designated as the $\mathrm{M}$ profile below) is based on a general circulation model (GCM) simulation from Showman et al. (2009) smoothly connected to an upper atmosphere escape model (Yelle 2004). The Line et al. (2014) profile is retrieved from secondary eclipse observations. These authors report a $p-T$ profile in the range between 1 mbar and $10 \mathrm{bar}$, with the retrieval primarily sensitive to the pressure range $10-100$ mbar. In order to extrapolate to other pressures in our calculations, we assumed either an isothermal behavior in both higher and lower pressure regimes (designated as the $\mathrm{L}$ profile), or an isotherm profile at higher pressures and a smooth connection to the escape calculations of Koskinen et al. (2013) for the upper atmosphere (designated as LK). We also consider another temperature profile $(\mathrm{MK})$ that utilizes the cooler temperature $p-T$ profile from the GCM calculations smoothly connected to the thermal structure of the upper atmosphere calculated by Koskinen et al. (2013). The MK temperature profile is in better agreement with the skin temperatures anticipated for this atmosphere (Figure 3). Finally, for the comparison with HD $209458 \mathrm{~b}$, we assume the corresponding day average $p-T$ profile from Moses et al. (2011) for this atmosphere. For the atmospheric mixing, we use the $K_{\mathrm{ZZ}}$ profiles suggested by the GCMs for the two planets, but we perform a sensitivity test for the $0.1 \times K_{\mathrm{ZZ}}$ and $0.01 \times K_{\mathrm{ZZ}}$ profiles. Below we discuss the results for the chemical composition and estimates of the aerosol mass fluxes, the resulting properties of the formed aerosols, and their impact on the planetary transit.

\subsection{Photochemical Mass Fluxes}

In order to evaluate the significance of aerosol production, we first compare the mass fluxes generated by the photolysis of major compounds in the atmospheres of HD $209458 \mathrm{~b}$ and HD 
Table 1

Mass Fluxes (in $\left.\mathrm{g} \mathrm{cm}^{-2} \mathrm{~s}^{-1}\right)$ Produced from the Photolysis of Different Species in the Upper Atmospheres $\left(p \leqslant 10^{-5}\right.$ bar) of HD $209458 \mathrm{~b}$ and HD 189733 b Under Different Assumptions for the Thermal Structure $\left(a(b)=a \times 10^{b}\right)$

\begin{tabular}{|c|c|c|c|c|c|c|}
\hline \multirow[b]{3}{*}{ Species } & \multicolumn{6}{|c|}{ HD $189733 \mathrm{~b}$} \\
\hline & \multicolumn{3}{|c|}{ M } & \multicolumn{3}{|c|}{ MK } \\
\hline & $K_{\mathrm{ZZ}}$ & $0.1 \times K_{\mathrm{ZZ}}$ & $0.01 \times K_{\mathrm{ZZ}}$ & $K_{\mathrm{ZZ}}$ & $0.1 \times K_{\mathrm{ZZ}}$ & $0.01 \times K_{\mathrm{ZZ}}$ \\
\hline $\mathrm{CH}_{4}$ & $8.1(-14)$ & $7.1(-17)$ & $1.9(-18)$ & $7.1(-16)$ & $2.2(-19)$ & $3.7(-21)$ \\
\hline $\mathrm{HCN}$ & $6.4(-11)$ & $2.8(-11)$ & $2.6(-12)$ & $2.9(-11)$ & $2.0(-12)$ & $6.8(-14)$ \\
\hline $\mathrm{C}_{2} \mathrm{H}_{2}$ & $3.3(-11)$ & $6.5(-15)$ & $1.9(-19)$ & $1.8(-11)$ & $8.1(-16)$ & $4.0(-18)$ \\
\hline $\mathrm{CO}$ & $1.5(-12)$ & $2.3(-13)$ & $5.0(-16)$ & $4.8(-12)$ & $2.0(-12)$ & $4.9(-15)$ \\
\hline $\mathrm{N}_{2}$ & $8.3(-13)$ & $4.2(-13)$ & $6.0(-12)$ & $7.0(-13)$ & $3.3(-13)$ & $4.2(-14)$ \\
\hline $\mathrm{NH}_{3}$ & $1.2(-15)$ & $1.9(-16)$ & $2.7(-15)$ & $1.6(-15)$ & $4.4(-17)$ & $8.3(-18)$ \\
\hline $\mathrm{H}_{2} \mathrm{~S}$ & $3.0(-14)$ & $9.1(-15)$ & $2.1(-14)$ & $5.9(-13)$ & $9.3(-14)$ & $5.6(-14)$ \\
\hline $\mathrm{S}_{3}$ & $6.8(-12)$ & $2.5(-12)$ & $8.5(-11)$ & $1.4(-12)$ & $4.0(-13)$ & $3.0(-13)$ \\
\hline \multirow[t]{2}{*}{ Soot $(1 \%)$} & $9.7(-13)$ & $2.8(-13)$ & $2.6(-14)$ & $4.7(-13)$ & $2.0(-14)$ & $6.8(-16)$ \\
\hline & \multicolumn{3}{|c|}{$\mathrm{L}$} & \multicolumn{3}{|c|}{ LK } \\
\hline Species & $K_{\mathrm{ZZ}}$ & $0.1 \times K_{\mathrm{ZZ}}$ & $0.01 \times K_{\mathrm{ZZ}}$ & $K_{\mathrm{ZZ}}$ & $0.1 \times K_{\mathrm{ZZ}}$ & $0.01 \times K_{\mathrm{ZZ}}$ \\
\hline $\mathrm{CH}_{4}$ & $1.0(-15)$ & $4.6(-20)$ & $1.6(-22)$ & $2.7(-16)$ & $1.3(-19)$ & $2.1(-21)$ \\
\hline $\mathrm{HCN}$ & $2.9(-11)$ & $1.4(-11)$ & $7.7(-15)$ & $2.2(-12)$ & $2.9(-13)$ & $1.2(-14)$ \\
\hline $\mathrm{C}_{2} \mathrm{H}_{2}$ & $1.5(-15)$ & $9.7(-19)$ & $1.9(-22)$ & $3.0(-14)$ & $5.5(-16)$ & $4.3(-18)$ \\
\hline $\mathrm{CO}$ & $8.5(-13)$ & $3.3(-13)$ & $4.7(-17)$ & $5.1(-12)$ & $2.3(-12)$ & $6.1(-15)$ \\
\hline $\mathrm{N}_{2}$ & $9.7(-13)$ & $4.1(-13)$ & $6.3(-12)$ & $7.7(-13)$ & $3.7(-13)$ & $6.1(-12)$ \\
\hline $\mathrm{NH}_{3}$ & $8.2(-16)$ & $7.0(-18)$ & $1.6(-16)$ & $6.4(-16)$ & $6.0(-18)$ & $1.5(-16)$ \\
\hline $\mathrm{H}_{2} \mathrm{~S}$ & $4.0(-13)$ & $1.1(-13)$ & $9.0(-14)$ & $5.0(-13)$ & $1.2(-13)$ & $9.2(-14)$ \\
\hline $\mathrm{S}_{3}$ & $1.6(-13)$ & $5.0(-14)$ & $4.3(-14)$ & $1.5(-13)$ & $5.0(-14)$ & $4.3(-14)$ \\
\hline \multirow[t]{2}{*}{ Soot $(1 \%)$} & $2.9(-13)$ & $1.4(-13)$ & $7.7(-17)$ & $2.2(-14)$ & $2.9(-15)$ & $1.2(-16)$ \\
\hline & & \multicolumn{5}{|c|}{ HD 209458 b } \\
\hline Species & & $K_{\mathrm{ZZ}}$ & & $0.1 \times K_{\mathrm{ZZ}}$ & & $0.01 \times K_{\mathrm{ZZ}}$ \\
\hline$\overline{\mathrm{CH}_{4}}$ & & $1.1(-22)$ & & $7.5(-25)$ & & $4.7(-25)$ \\
\hline $\mathrm{HCN}$ & & $7.8(-15)$ & & $2.3(-15)$ & & $3.2(-15)$ \\
\hline $\mathrm{C}_{2} \mathrm{H}_{2}$ & & $2.8(-17)$ & & $1.2(-18)$ & & $2.3(-18)$ \\
\hline $\mathrm{CO}$ & & $4.8(-11)$ & & $3.0(-11)$ & & $1.7(-11)$ \\
\hline $\mathrm{N}_{2}$ & & $4.5(-12)$ & & $2.2(-12)$ & & $9.9(-13)$ \\
\hline $\mathrm{NH}_{3}$ & & $3.5(-16)$ & & $9.3(-17)$ & & $9.2(-17)$ \\
\hline $\mathrm{H}_{2} \mathrm{~S}$ & & $1.8(-11)$ & & $7.2(-12)$ & & $6.8(-12)$ \\
\hline $\mathrm{S}_{3}$ & & $1.8(-19)$ & & $9.2(-20)$ & & $9.0(-20)$ \\
\hline Soot $(1 \%)$ & & $7.8(-17)$ & & $2.3(-17)$ & & $3.2(-17)$ \\
\hline
\end{tabular}

$189733 \mathrm{~b}$, under the different thermal structures assumed (Table 1). The mass flux from the photolysis of methane, or of its primary products $\left(\mathrm{HCN}, \mathrm{C}_{2} \mathrm{H}_{2}\right)$, is significantly larger in the atmosphere of HD 189733 b relative to HD 209458 b. In both atmospheres and under all assumed temperature profiles, methane is destroyed near $\sim 1$ mbar and is replaced primarily by $\mathrm{HCN}$ and secondarily by $\mathrm{C}_{2} \mathrm{H}_{2}$. However, the abundance of these species surviving in the upper atmosphere is significantly larger for HD 189733 b compared to HD 209458 b (Figure 4). This difference is the result of the rapid temperature increase at $p<10$ mbar in HD 209458 b (Figure 3) that drives the atmospheric chemical equilibrium closer to the thermochemical limit, i.e., the carbon balance is efficiently shifted toward $\mathrm{CO}$ and the $\mathrm{CH}_{4}$ abundance surviving in the upper atmosphere is significantly reduced on HD 209458 b compared to HD 189733 b. In practical terms, the combined mass flux from the photolysis of $\mathrm{CH}_{4}$ and its secondary products is between 2 and 4 orders of magnitude smaller in HD 209458 b relative to that of HD $189733 \mathrm{~b}$, depending on the assumed temperature profile for the latter atmosphere. Thus, we note that the thermal structure in the transition between the lower and upper atmospheres (practically the pressure range between 1 mbar and $1 \mu$ bar) has a major impact on the atmospheric composition from the point of view of complex chemical products, and current observations do not provide solid constraints for this parameter on either planet.

The large differences observed between the two planets considered should not obscure the sensitivity of the composition results to the assumed temperature profiles for the HD 189733 b atmosphere. Evidently, the large temperature difference between the simulated profile $(\mathrm{M})$ and the retrieved profile (L) in the lower atmosphere (more than $1000 \mathrm{~K}$; see Figure 3 ) results in changes to the chemical composition of the whole atmosphere (Figure 4), which could affect the primary and secondary eclipse observations if the accuracy of the measurements is improved in the near future. This variability is also evident in the calculated mass fluxes (Table 1). The total mass flux from the photolysis of $\mathrm{CH}_{4}, \mathrm{C}_{2} \mathrm{H}_{2}$, and $\mathrm{HCN}$ ranges between $2.2 \times 10^{-12}$ and $9.7 \times 10^{-11} \mathrm{~g} \mathrm{~cm}^{-2} \mathrm{~s}^{-1}$ among the four temperature profiles considered. The relative variation for the individual molecules can be much larger. Assuming a $1 \%$ efficiency for aerosol formation results in aerosol mass fluxes of the order of $10^{-14}-10^{-12} \mathrm{~g} \mathrm{~cm}^{-2} \mathrm{~s}-1$, which are large enough to have important ramifications for the resulting aerosol opacity. The question arising from this comparison is, why are the simulated and retrieved conditions 

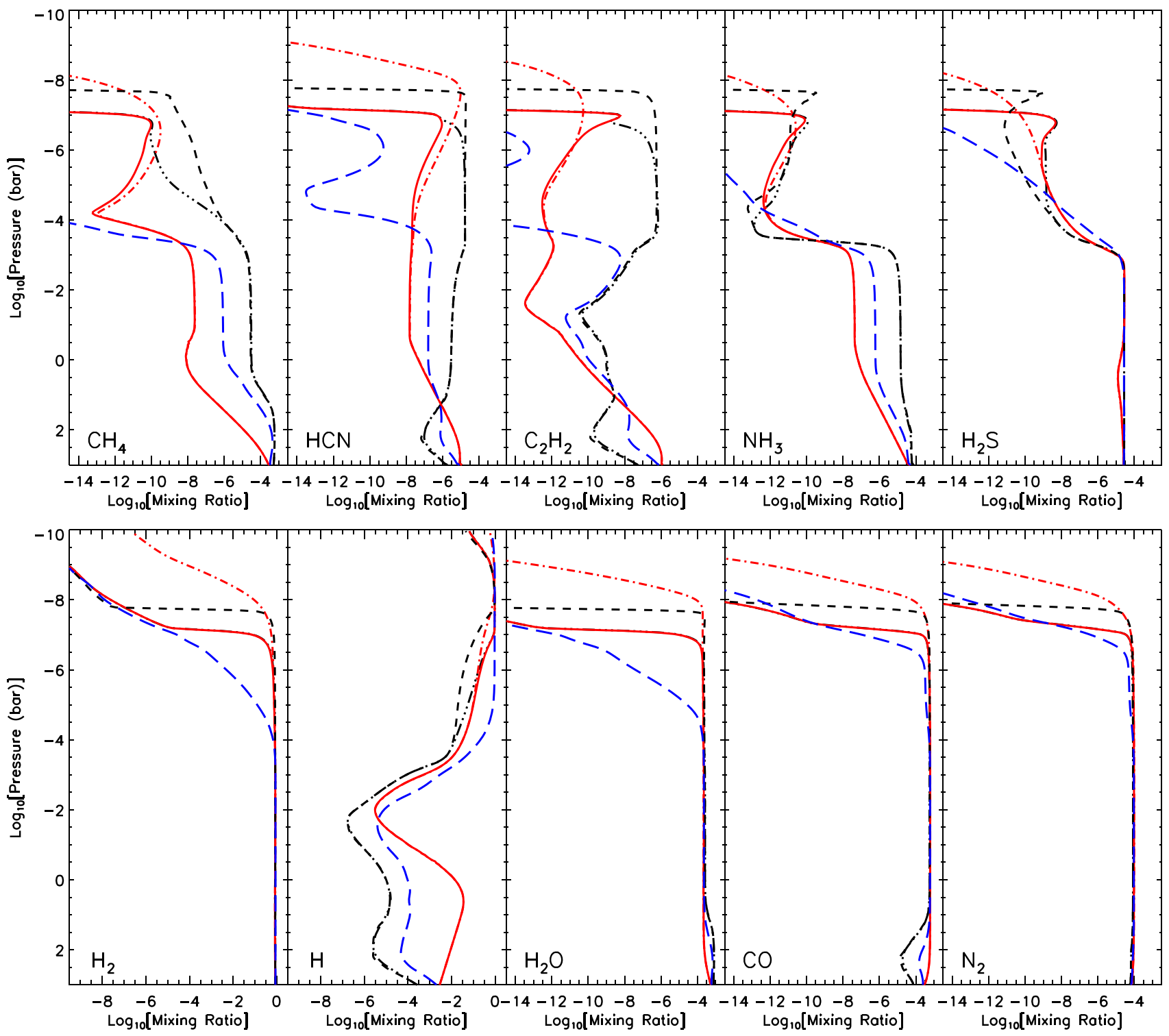

Figure 4. Mixing ratio profiles of major species for the different temperature profiles assumed for HD $2093458 \mathrm{~b}$ and HD $189733 \mathrm{~b}$. We assumed the nominal $K_{\mathrm{ZZ}}$ profile for this simulation. Line styles and colors correspond to the temperature profiles of Figure 3, i.e., red solid: LK profile, red dashed-dotted: L profile, black dashed: M profile, black dashed-triple-dotted: MK profile, blue long-dashed: HD 209458 b's profile.

so different and how could the simulated $p-T$ profile be brought in better agreement with the observations? Could the heating by the anticipated hazes help in this aspect? We evaluate this scenario further below.

We further note that the differences in the thermal structure assumed in the upper atmosphere of HD $189733 \mathrm{~b}$ have further ramifications for the physical processes taking place there. Comparison of the model results between the (M, MK) or the (L, LK) profiles demonstrate how the composition is modified by assuming changes only in the thermal structure of the upper atmosphere (see Figure 3). Lower temperatures allow for the survival of species to higher altitudes (lower pressures). This characteristic is particularly evident when comparing the $\mathrm{L}$ profile with the LK profile where the latter includes a hot thermosphere suggested by atmospheric escape models. The escape models indicate that the temperature should increase within a few atmospheric scale heights as soon as the dominance of $\mathrm{H}_{2}$ is overthrown by the increasing abundance of atomic hydrogen (Yelle 2004; García Muñoz 2007;
Koskinen et al. 2007, 2013), which is formed through the catalytic destruction of $\mathrm{H}_{2}$ by $\mathrm{OH}$ (Moses et al. 2011), as well as by $\mathrm{O}$ and $\mathrm{S}$ (which in turn are produced from the destruction of their parent molecules, $\mathrm{H}_{2} \mathrm{O}$ and $\mathrm{H}_{2} \mathrm{~S}$ ). The increasing $\mathrm{H}$ abundance has its own ramifications for the abundances of different species, which depend on the chemical pathways defining the chemical equilibrium for each case, but which in general leads to the destruction of molecular structures (Figure 4). At lower temperatures, the $\mathrm{H}_{2}$ to $\mathrm{H}$ transition is slower, thus molecular species can survive at higher altitudes. As the production of photochemical aerosols depends critically on the production of large molecular structures, the temperature assumed in the transition between the lower atmosphere and the thermosphere can play an important role in the nature and abundance of photochemical aerosols.

Apart from the temperature, assumptions on the eddy mixing profile have an impact on the simulated composition profiles, and therefore on the resulting aerosol mass fluxes. We consider as the nominal mixing profile for each planet case the profiles 
presented in Moses et al. (2011), which are based on GCM simulations (Showman et al. 2009). Subsequent studies suggested that the actual atmospheric mixing efficiencies could be lower depending on the way the eddy diffusivity is calculated based on the GCM simulations (Parmentier et al. 2013). In order to evaluate the possible influence of reduced atmospheric mixing, we also performed calculations with $10 \times$ and $100 \times$ reduced eddy profiles relative to the nominal case (see Table 1). This evaluation demonstrates that with reduced atmospheric mixing, the simulated mass fluxes are reduced as anticipated; for $\mathrm{CH}_{4}$, the reduced eddy profile allows its abundance to remain close to the thermochemical equilibrium solution at lower pressures than those for the nominal eddy profile case, thus resulting in a smaller methane abundance in the upper atmosphere. On the other hand, the mixing ratios of $\mathrm{CO}, \mathrm{N}_{2}$ and $\mathrm{H}_{2} \mathrm{O}$ are controlled by thermochemical equilibrium at high pressures and remain constant in the remainder of the atmosphere before diffusive separation decreases their abundance in the upper atmosphere (Moses et al. 2011). Thus, reduced mixing for these species lowers their homopause altitude, decreasing in this way their abundance in the upper atmosphere. However, for all cases considered, HCN remains the most abundant hydrocarbon in the upper atmosphere and its photolysis provides significant mass fluxes for the formation of hazes.

Finally, other species also generate significant mass fluxes of higher-order compounds. For example, the chemistry of $\mathrm{H}_{2} \mathrm{~S}$ leads to the formation of polysulfur compounds, from which we track species up to $S_{3}$ in these simulations. The mass flux from the photolysis of $\mathrm{S}_{3}$ is also significant, ranging between $\sim 10^{-13}$ and $\sim 7 \times 10^{-12} \mathrm{~g} \mathrm{~cm}^{-2} \mathrm{~s}^{-1}$ for the different temperature profiles assumed on HD $189733 \mathrm{~b}$, while it is more than five orders of magnitude smaller for the atmosphere of HD 209458 b. However, the high-temperature conditions do not allow the sulfur compounds to condense in the simulated atmospheres.

\subsection{Aerosol Properties}

With the estimated mass fluxes, we can evaluate the resulting properties of the aerosol particle distribution. We present simulations only for the $\mathrm{M}$ and $\mathrm{L}$ temperature profiles as the other cases only correspond to changes in the upper atmosphere that will not significantly modify the results of the aerosol microphysics occurring at lower altitudes, unless the temperature increases rapidly enough to affect the ablation of the aerosol particles. Figure 5 presents an overview of our results in terms of the average particle size and corresponding density under different assumptions of the particle mass flux generated by the photochemistry, the eddy mixing efficiency, and the atmospheric thermal structure of HD 189733 b and HD 209458 b. For these simulations, we assume that the initial particles formed by photochemistry have a typical radius of $1 \mathrm{~nm}$; therefore, a given mass flux translates to the production rate of particles in the first bin of our aerosol grid. As soon as they are formed, particles start to coagulate and grow in size, forcing the particle population to decrease. The efficiency of particle coagulation depends directly on the particle thermal velocity, i.e., the temperature, and the sticking efficiency of the particles (which for the moment we assume is 1 for this evaluation, i.e., no charge effects).

The differences in the two thermal profiles we consider for the upper atmosphere of HD 189733 b cause only small
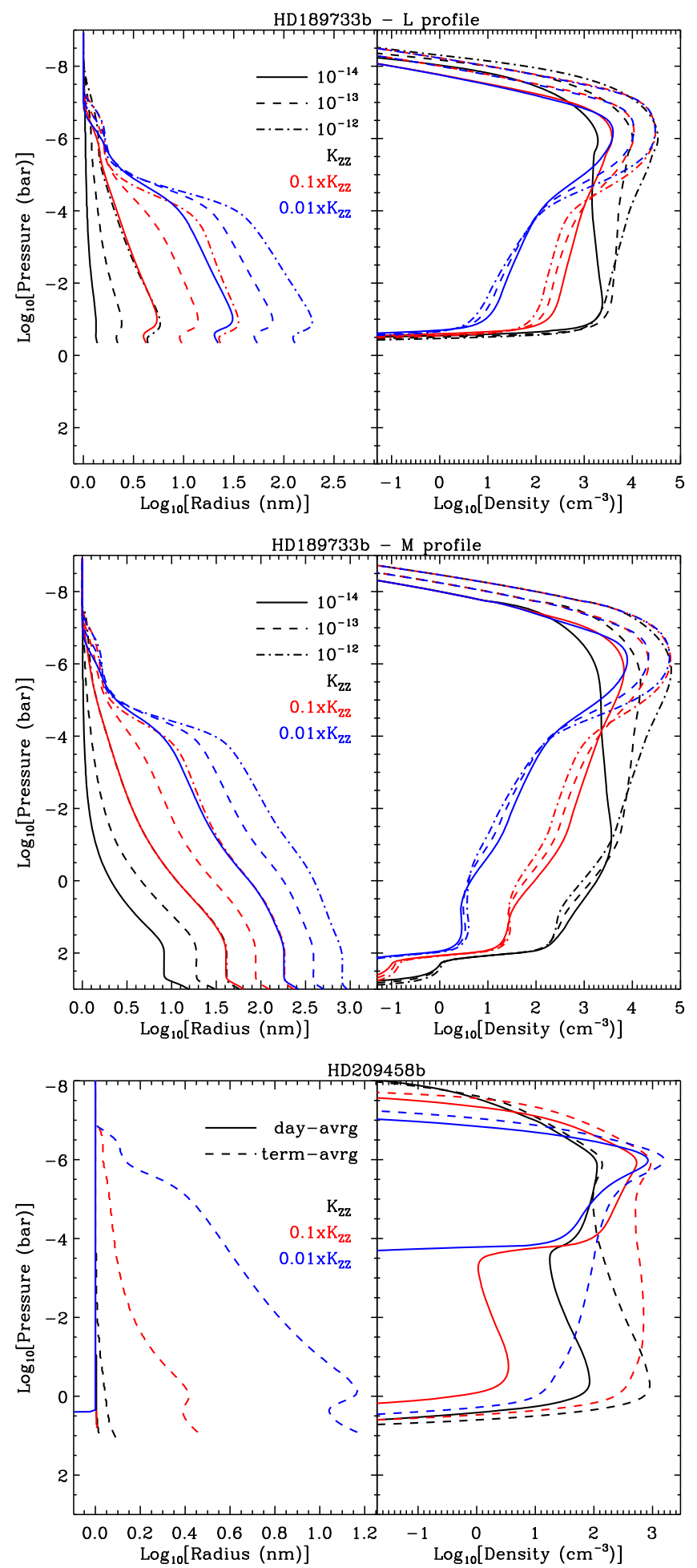

Figure 5. Average aerosol particle radius and corresponding density, under different assumptions of the aerosol mass flux (line style) and atmospheric mixing (colors). The first two panels present the results for the case of HD $189733 \mathrm{~b}$ for the $\mathrm{L}$ and $\mathrm{M}$ temperature profiles, while the last panel presents the model results for the case of HD $209458 \mathrm{~b}$.

variations in the particle coagulation rates (which scale as the square root of the temperature), and thus the resulting average particle size and density are similar for the corresponding mass fluxes and eddy profiles (Figure 5). However, the temperature structure assumed in the lower atmosphere (below $\sim 10$ mbar) has major implications for the resulting particle properties. For 
the $\mathrm{L}$ profile, assuming a soot-type composition, particles thermally decompose below 0.1 bar, leading to their rapid loss, as demonstrated by the particle density profiles (Figure 5). On the contrary, for the cooler $\mathrm{M}$ profile, particle decomposition occurs for $p>100$ bar, and particles can survive to deeper pressure levels in the atmosphere, where they grow to larger sizes. Assuming a different chemical composition for the photochemical aerosols results in particle decomposition at cooler temperatures, i.e., at even lower pressures than for the soot-composition case.

The average particle radius depends on the mass flux assumed as this parameter defines the population of particles available for collisions, with larger values resulting in larger particles: for the $\mathrm{L}$ profile, in the $10^{-12} \mathrm{~g} \mathrm{~cm}^{-2} \mathrm{~s}^{-1}$ mass flux case, particles grow up to $\sim 6 \mathrm{~nm}$; in the $10^{-13} \mathrm{~g} \mathrm{~cm}^{-2} \mathrm{~s}^{-1}$ case, the average size reaches $2.5 \mathrm{~nm}$; while for the $10^{-14} \mathrm{~g} \mathrm{~cm}^{-2} \mathrm{~s}^{-1}$ case, particle coagulation is practically inefficient and the particle density profile demonstrates the effect of rapid transport from the formation region to the lower atmosphere where their population increases due to the slower vertical transport. For the $\mathrm{M}$ profile, aerosols grow further in the lower atmosphere, reaching maximum average radii of 8,20 , and $40 \mathrm{~nm}$ for the three mass flux cases considered.

Particle vertical transport has an indirect effect on their coagulation, which dominates over the direct temperature influence. If particles are transported faster than they collide at a given pressure level, their coagulation will be limited and thus their size will remain approximately constant until they reach a pressure region where collisions are efficient. Comparison of the model results at different eddy mixing efficiencies demonstrates this effect: for the nominal $K_{\mathrm{ZZ}}$ atmospheric transport limits, the collisions of the formed particles and the resulting average particle size remains small and only increases in the deep atmosphere as the eddy mixing is reduced. Under the reduced atmospheric mixing conditions, collisions are more frequent and particles grow rapidly below their production region. For the $\mathrm{L}$ profile, particles reach average radii of $\sim 35 \mathrm{~nm}$ and $\sim 200 \mathrm{~nm}$ for the $0.1 \times K_{\mathrm{ZZ}}$ and $0.01 \times K_{\mathrm{ZZ}}$ cases, respectively, under the highest mass flux case considered $\left(10^{-12} \mathrm{~g} \mathrm{~cm}^{-2} \mathrm{~s}^{-1}\right)$. The corresponding radii for the $\mathrm{M}$ case are $\sim 185 \mathrm{~nm}$ and $\sim 750 \mathrm{~nm}$. Rapid coagulation decreases the corresponding particle densities below their formation region and results in progressively smaller densities in the lower atmosphere for the higher mass flux assumed (Figure 5). A similar effect would result from the planet gravity field with larger-mass planets pulling particles more efficiently, thereby reducing their growth, under otherwise similar temperature, particle mass flux, and atmospheric mixing conditions.

The average particle properties for the atmosphere of HD 209458 b are strongly affected by ablation as the rapid temperature increase above the 10 mbar pressure level results in the partial decomposition of the particles formed at higher altitudes (Figure 5). To further demonstrate the small contribution that photochemical hazes should have to the opacity of this atmosphere, we consider an aerosol mass flux of $10^{-15} \mathrm{~g} \mathrm{~cm}^{-2} \mathrm{~s}^{-1}$, which corresponds to a $\sim 10 \%$ efficiency of photochemical aerosol formation for the largest photochemical mass fluxes generated according to Table 1. For both the nominal and the reduced atmospheric mixing profiles, the formed particles do not grow and only the population of the first radius bin is modified depending on how efficiently particles are moved away from parts of the atmosphere where they can decompose. We also evaluated the resulting aerosol properties assuming the terminator average thermal structure reported by Moses et al. (2011). This scenario assumes that the formed photochemical aerosols are rapidly transported horizontally from their formation region to the limbs of the planet where the low temperatures allow for their survival. Under this assumption, particles sustain a larger population but their size does not significantly increase as the stronger atmospheric mixing for this planet limits their collision for either the nominal or reduced eddy profiles. Thus, for HD 209458 b, both the lower anticipated mass fluxes as well as the high temperature and strong atmospheric mixing limit the abundance of photochemical aerosols in its atmosphere. As the observational constraints for the thermal structure of HD 209458 b's upper atmosphere (above 1 mbar) are limited, we can inversely argue that the temperature in the upper atmosphere of this planet must be hotter than that on HD $189733 \mathrm{~b}$ in order to explain the lack of significant hazes in the primary transit observations.

\subsection{Transit Depth}

With the simulated aerosol distribution and the corresponding chemical composition from the photochemical model, we can evaluate the simulated transit depth for each case. We calculate the aerosol extinction at each altitude using Mie theory (spherical particles) and the refractive index for soot particles (the details of this parameter are discussed below). For the gas components, we consider Rayleigh scattering by $\mathrm{H}_{2}$, collision-induced absorption by $\mathrm{H}_{2}-\mathrm{H}_{2}$ and $\mathrm{H}_{2}-\mathrm{He}$ pairs, extinction by $\mathrm{Na}$ and $\mathrm{K}$, and absorption by $\mathrm{H}_{2} \mathrm{O}, \mathrm{CO}$, and $\mathrm{CH}_{4}$. We calculate molecular lines using the high-temperature line lists of Exomol (Tennyson et al. 2016) and Theorets (Rey et al. 2016). For the planet size of HD $189733 \mathrm{~b}$, we use the reported $R_{P} / R_{S}$ values from Pont et al. (2013), which are consistent with the latest review (Sing et al. 2016). We calculate the transit depth by evaluating the attenuation of the stellar flux over the whole atmosphere (Brown 2001), and we use planet and star parameters from Boyajian et al. (2014).

There is a well-known degeneracy in the calculation of the planet size for gas giants due to the inability to know the atmospheric pressure at the observed planet size. In order to have a consistent picture among the different cases we evaluate, we normalize our simulated spectra to the reported planet size at the $8 \mu \mathrm{m}$ band from Spitzer/IRAC observations. These observations are believed to be less affected by stellar limb effects, and they demonstrate the smallest uncertainties of all near-IR observations (Agol et al. 2010). To perform the normalization, we assume an initial reference pressure level for the reported $R_{P} / R_{S}$, and with the corresponding altitude grid for the assumed temperature profile (calculated hydrostatically), we calculate the transit depth and the corresponding pressure probed at each wavelength. We then evaluate the average pressure in the $8 \mu \mathrm{m}$ IRAC band, taking into account the instrument response within the band (Hora et al. 2008), and repeat the transit calculation assuming that the reported planet size at the $8 \mu \mathrm{m}$ band corresponds to this average pressure. Thus, all simulated spectra are identical at the $8 \mu \mathrm{m}$ band, but the reference pressure for each case is different, reflecting the changes in the gaseous and aerosol opacities for each case.

We assume that the photochemical lifetimes are much smaller than the characteristic times for the evolution of the 


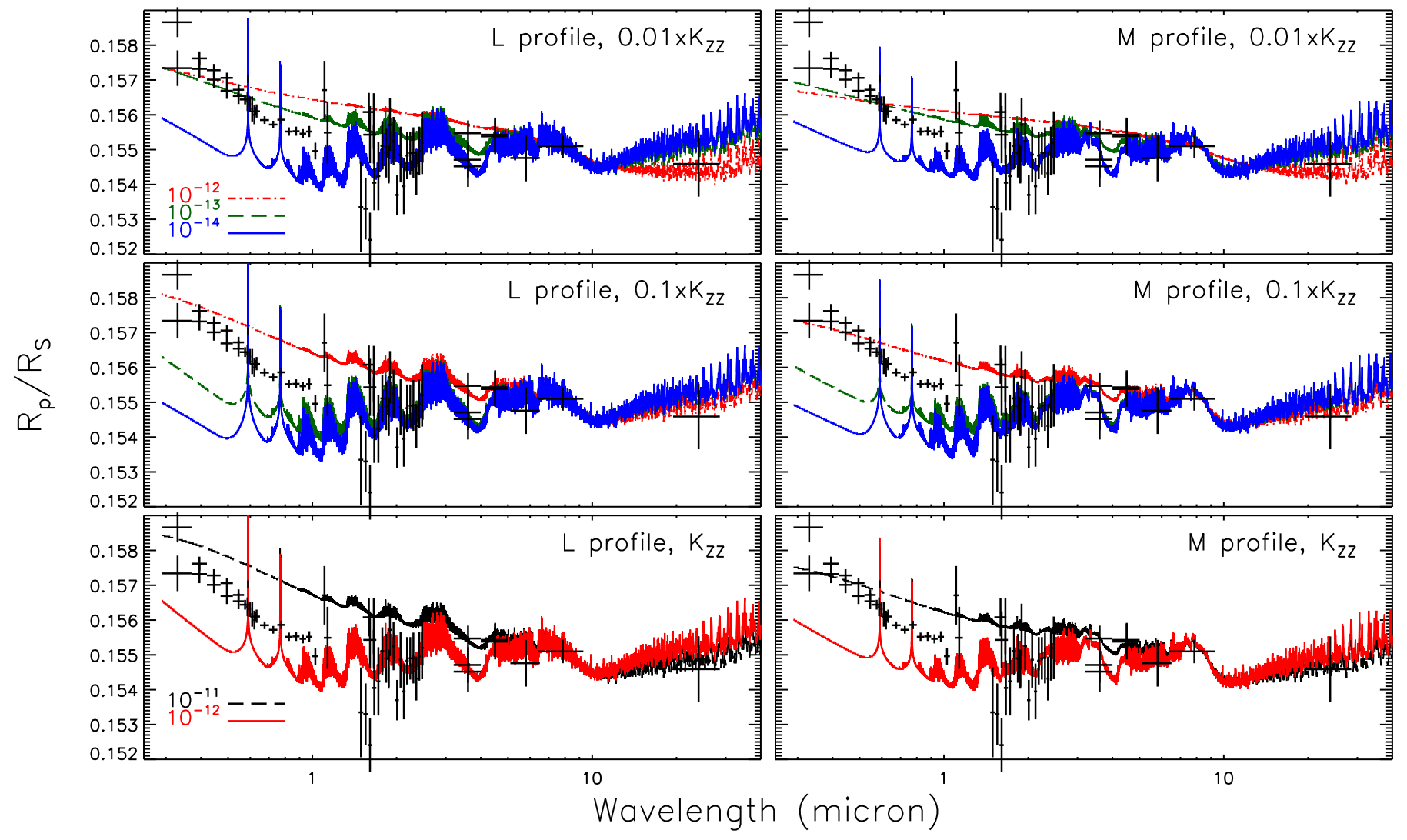

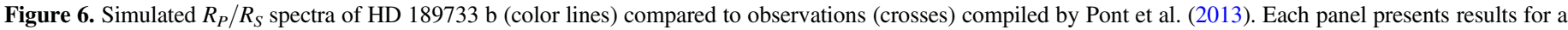

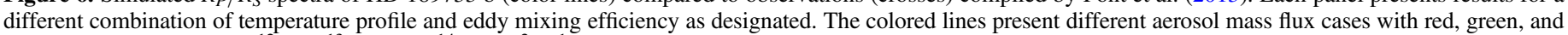
blue corresponding to $10^{-12}, 10^{-13}$, and $10^{-14} \mathrm{~g} \mathrm{~cm}^{-2} \mathrm{~s}^{-1}$, respectively.

microphysical aerosol population, i.e., the mass flux of aerosols is driven by the conditions at high illumination and once the particles are formed, they will be horizontally distributed. In contrast, the gaseous composition will be modified locally due to the changes in the solar insolation. Thus, for the transit calculations, we use chemical composition profiles that we calculate assuming limb-geometry conditions for the photolysis rates (examples of limb profiles are presented below). Temperature variations between the limbs and the disk can also affect the resulting transit size (Line \& Parmentier 2016). However, we focus here on the differences incurred by the temperature profiles we consider, which demonstrate larger variations than those anticipated from the disk-limb variations. For example, the lower temperatures suggested by the $\mathrm{M}$ profile in the lower atmosphere allow for a higher abundance of $\mathrm{CH}_{4}$ to survive in the upper atmosphere. This results in clear signatures in the simulated transit spectra near the strong $\nu_{3}$ $(3.3 \mu \mathrm{m})$ and $\nu_{4}(7.7 \mu \mathrm{m})$ methane bands, which are missing in the warmer $\mathrm{L}$ profile case that is dominated by $\mathrm{H}_{2} \mathrm{O}$ absorption. Current observations do not resolve these spectral regions, but future measurements with JWST should be able to identify such features, if present. Nevertheless, the overall picture of the transit spectra for these two extreme profiles is rather similar and only the reference pressure level changes between the two cases.

The impact of the simulated aerosol distribution on the planet size is mainly affecting the UV and visible part of the spectrum, as anticipated by the small particle radius (Figure 6). The resulting $R_{P} / R_{S}$ spectra demonstrate how the planet size increases at short wavelengths as the assumed aerosol mass flux increases. The simulated spectra clearly show the inverse relationship between the aerosol mass flux and the atmospheric eddy mixing, with lower mixing requiring smaller aerosol mass fluxes to match the observations. For the nominal eddy profile, mass fluxes between $10^{-12}$ and $10^{-11} \mathrm{~g} \mathrm{~cm}^{-2} \mathrm{~s}^{-1}$ bracket the observations for both temperature profiles ( $\mathrm{L}$ and $\mathrm{M}$ ), while for weaker atmospheric mixing, the required mass flux is smaller and a flux of $10^{-12} \mathrm{~g} \mathrm{~cm}^{-2} \mathrm{~s}^{-1}$ provides an upper bound. This effect results from the larger particle radius accessible for the low atmospheric mixing efficiencies, which allows for an increase of the particle cross-section that outbalances the drop in the corresponding particle density (see Figure 5). However, the particle radius modifies the slope of the particle extinction at short wavelengths, which defines the shape of the simulated spectra. Small particles lead to steep slopes that progressively become shallower as the particle radius increases. This trend is obvious in the simulated spectra, particularly for the lowest eddy case $\left(0.01 \times K_{\mathrm{ZZ}}\right)$, which provides the largest particle radii $(\sim 10 \mathrm{~nm}$ at the probed pressures). In this case, the simulated transit spectrum has a much shallower slope at short wavelengths compared to the nominal eddy case for which the particle radius remains small $(\sim 1-2 \mathrm{~nm}$ at the probed pressures).

If we attempt to fit the observation with the current assumptions, we find that for the lowest eddy case $\left(0.01 \times K_{\mathrm{ZZ}}\right)$ a mass flux of the order of $6-7 \times 10^{-14} \mathrm{~g} \mathrm{~cm}^{-2} \mathrm{~s}^{-1}$ is required (Table 2). Such a mass flux implies a photochemical aerosol production efficiency of $\sim 0.1 \%-0.2 \%$ relative to the mass fluxes involved in soot formation (Table 1). The other atmospheric mixing cases 
Table 2

Best Solutions in Terms of Required Aerosol Mass Flux for Each Temperature and Eddy Case Considered

\begin{tabular}{|c|c|c|c|c|c|c|c|c|c|}
\hline Profile & $\begin{array}{l}\text { Eddy } \\
\left(K_{\mathrm{ZZ}}\right)\end{array}$ & $\begin{array}{l}\text { Mass Flux } \\
\left(\mathrm{g} \mathrm{cm}^{-2} \mathrm{~s}^{-1}\right)\end{array}$ & $\begin{array}{c}\epsilon \\
(\%)\end{array}$ & $\begin{array}{c}P_{\text {ref }} \\
\text { (mbar) }\end{array}$ & $\chi^{2}$ & $\begin{array}{l}r_{300} \\
(\mathrm{~nm})\end{array}$ & $\begin{array}{c}P_{300} \\
(\mu \text { bar })\end{array}$ & $\begin{array}{l}r_{500} \\
(\mathrm{~nm})\end{array}$ & $\begin{array}{c}P_{500} \\
(\mu \text { bar })\end{array}$ \\
\hline \multirow[t]{2}{*}{$\mathrm{L}$} & 1 & $3(-12)$ & 10.35 & 2.34 & 1.7 & 1.5 & 2.5 & 2.3 & 59 \\
\hline & 0.1 & $4(-13)$ & 2.85 & 2.28 & 1.8 & 1.7 & 3.8 & 4.7 & 48 \\
\hline \multirow[t]{2}{*}{ M } & 1 & $5(-12)$ & 5.15 & 1.06 & 2.5 & 1.4 & 0.9 & 1.8 & 11 \\
\hline & 0.1 & $5(-13)$ & 3.57 & 1.48 & 2.7 & 1.6 & 2.3 & 2.7 & 21 \\
\hline
\end{tabular}

Note. We also provide $\epsilon$, the implied the aerosol production efficiency relative to the mass fluxes provided in Table 1; $P_{\text {ref }}$, the reference pressure at the $8 \mu \mathrm{m}$ band; the reduced $\chi^{2}$; and the average particle radii and the probed pressures at 300 and $500 \mathrm{~nm}$.

imply a progressively larger efficiency increasing from $3 \%$ to $10 \%$ as the eddy is increased from $0.1 \times K_{\mathrm{ZZ}}$ to the nominal case. The required mass flux for the nominal eddy case $\left(3 \times 10^{-12} \mathrm{~g} \mathrm{~cm}^{-2} \mathrm{~s}^{-1}\right)$ is higher than that required for the $0.1 \times K_{\mathrm{ZZ}}$ case by a factor of $\sim 10$ for both temperature profiles (Table 2). Moreover, the reference pressures derived for the $\mathrm{L}$ profile are systematically higher than those for the $\mathrm{M}$ profile, which occur due to the increased contribution of $\mathrm{CH}_{4}$ that raises the total atmospheric opacity for the latter case.

The simulated spectra for the optimal solutions (in terms of their reduced $\chi^{2}$; see Table 2) demonstrate increasing planet size toward short wavelengths, while they indicate that solutions under the $\mathrm{L}$ temperature profile provide a slope that is closer to the observations relative to the $\mathrm{M}$ temperature profile, for both assumed eddy mixing cases (Figure 7). The residuals between model and observations are within $\sim 3 \sigma$ of the measurement uncertainty for the $\mathrm{L}$ case, while for the $\mathrm{M}$ case the model difference from the high-transit depth cases at short wavelengths approaches $\sim 4 \sigma$. The higher temperature at the probed pressures for the $\mathrm{L}$ case imposes a larger atmospheric scale height relative to the $M$ case, which increases the slope of the spectrum. Our results indicate that the average particle radius roughly doubles going from $\lambda=300 \mathrm{~nm}$ to $\lambda=500 \mathrm{~nm}$, while the probed pressures respectively increase from a few $\mu$ bar to $\sim 50 \mu$ bar (Table 2 ). At those pressures, we are near the aerosol production altitude, thus the assumptions made for the shape and location of the aerosol production profile can potentially affect the resulting average particle size and density, and therefore the simulated spectra at short wavelengths.

Another aspect that can affect the transit size at short wavelengths is the elemental composition, which we assume to be solar in the chemical composition simulation. Since the spectra are normalized to a common reference point (the $8 \mu \mathrm{m}$ IRAC band), modifying the mixing ratio of $\mathrm{H}_{2} \mathrm{O}$ while keeping all other components the same would affect the planet size at short wavelengths relative to the planet size in the visible to near-IR, which is dominantly affected by water absorption. A higher (lower) water abundance would cause light from the star to be attenuated at a higher (lower) altitude, and therefore bring the planet size in the IR closer to (farther away from) the planet size observed in the UV (see Figure 8). Thus, given the current uncertainty on the elemental composition of HD 189733 b's atmosphere and on the observed transit depth, it is pointless to seek for a better fit to the observations.

\section{Haze Interaction with the Atmosphere}

We now focus our attention on the possible implications that photochemical aerosols could have on the atmospheric structure and composition, and on how the particles interact with the gas-phase background. To perform this investigation, we need to know how the formed particles will interact with the radiation field. For this task, we used a compilation of laboratory investigations of the refractive index of soot particles (see Figure 9). These are based on the measurements of Chang \& Charalampopoulos (1990) and Lee \& Tien (1981) in the visible and IR, and the Gavilan et al. (2016) measurements in the UV. To check the consistency between the real and imaginary parts of the refractive index, we used the Krammers-Kroning dispersion relations. Thus, we derived average spectra of $n$ and $k$ from the available measurements (solid lines in Figure 9), from which we calculated the corresponding $k$ and $n$ through the dispersion formulae (dashed lines). The results suggest that the average $n$ and $k$ spectra are consistent with their corresponding measurements within the uncertainty of the derived laboratory values, which also rely on the dispersion formulae for their evaluations. Moreover, the observed differences translate to minor changes in the resulting extinction cross-sections of the aerosol particles (see Figure 9). Therefore, in the following we used the average $n$ and $k$ spectra (solid black lines).

\subsection{Radiation Field and Chemistry}

With the simulated aerosol particle distribution and crosssections, we can subsequently evaluate the contribution of aerosol particles to the atmospheric opacity. Taking as an example the case of the disk-average simulations for the $\mathrm{M}$ profile (see Figure 10) we can compare the total extinction optical depth due to the gaseous components with the corresponding opacity from the photochemical aerosols. The total aerosol optical depth is small compared to the total gaseous contribution in the deep atmosphere, while at short wavelengths $(\lambda<1000 \AA)$, absorption by hydrogen dominates the atmospheric opacity, and photons at those wavelengths are absorbed at altitudes above the region of the aerosol contribution (Figure 11). However, at longer wavelengths, the vertical distribution of aerosol particles provides a local opacity in the middle atmosphere ( $p<10$ mbar) that is larger than or comparable to the gaseous contribution. The importance of aerosol contribution varies across the wavelength range due to the strong variation of the gaseous opacity mainly from the alkali lines in the visible and from $\mathrm{H}_{2} \mathrm{O}$ absorption bands in the near-IR. Nevertheless, the presence of aerosols will affect both 

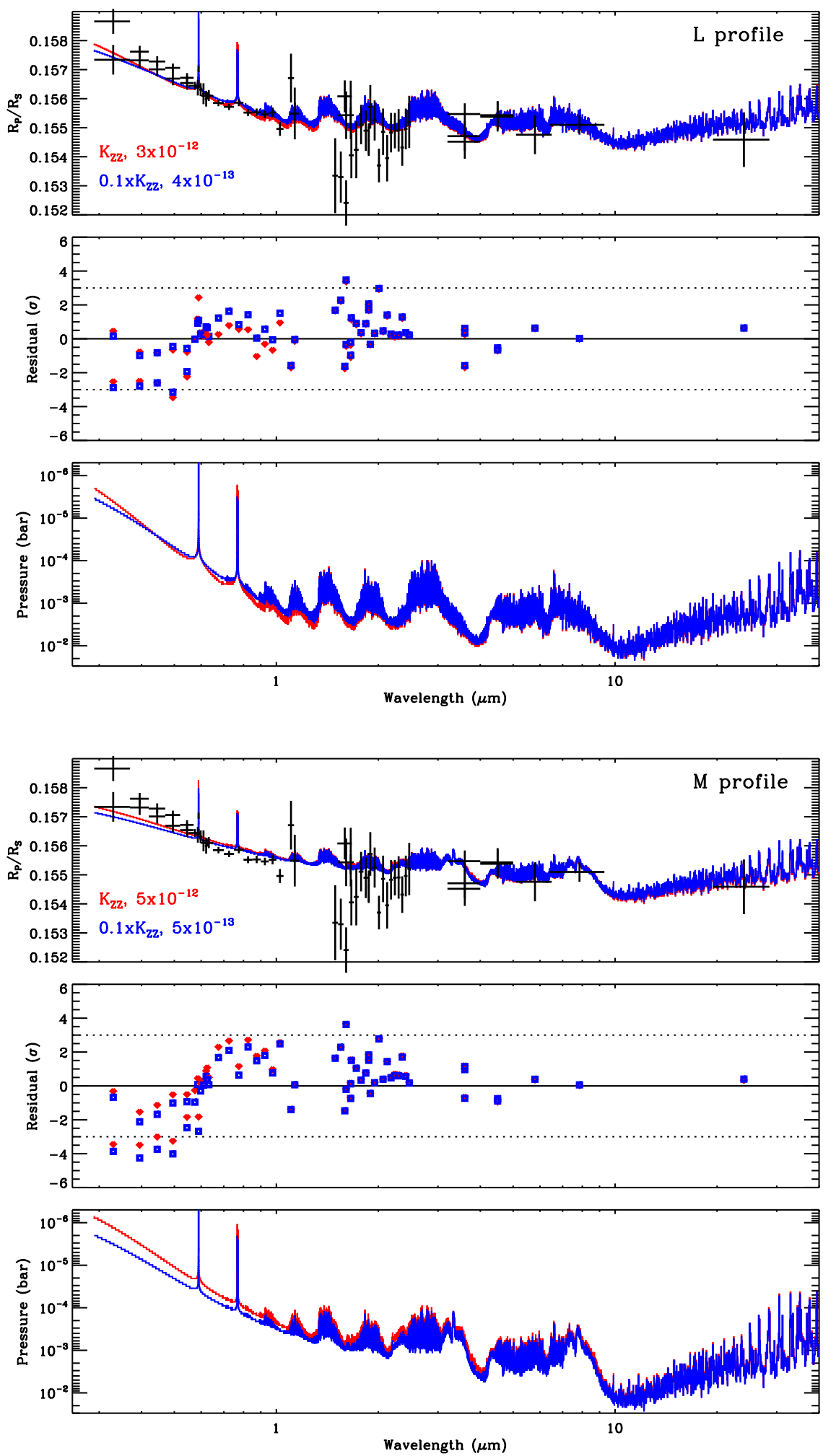

Figure 7. Planetary transit radius for an aerosol mass flux of $5 \times 10^{-13} \mathrm{~g} \mathrm{~cm}^{-2} \mathrm{~s}^{-1}$ (upper panel). The pink and cyan lines correspond to the $\mathrm{M}$ and $\mathrm{L}$ temperature profiles, respectively. The red and blue squares represent the model mapped onto the resolution of the observations. The latter are taken from the compilation of Pont et al. (2013). The simulated spectra are within $3 \sigma$ of the observations in the whole spectrum (middle panel), although they appear systematically lower than the observed planetary size at UV wavelengths. The two temperature profiles provide a similar transit spectrum, although the reference pressure at the $8 \mu \mathrm{m}$ Spitzer/IRAC band is smaller for the $\mathrm{M}$ profile relative to the $\mathrm{L}$ profile (lower panel).

the photolysis rates of different species as well as the local atmospheric heating.

Photons at wavelengths between 100 and $300 \mathrm{~nm}$ partake in photochemical processes of molecules larger than $\mathrm{H}_{2}$, therefore the modifications imposed by the aerosols affect the gaseous abundances. Our chemistry model results (Figure 12) demonstrate this effect when the simulated aerosol opacity is included in the evaluation of the atmospheric photolysis rates. These are 


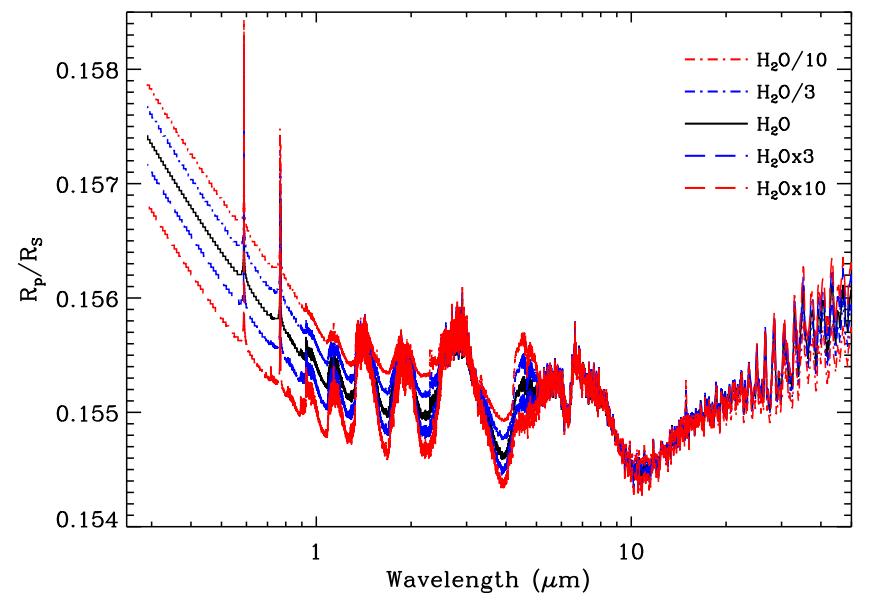

Figure 8. Simulated planet sizes assuming different $\mathrm{H}_{2} \mathrm{O}$ mixing ratios relative to solar abundances. Increasing the water abundance decrease the UV planet size, and conversely, decreasing the water abundance increases the contribution of aerosols.
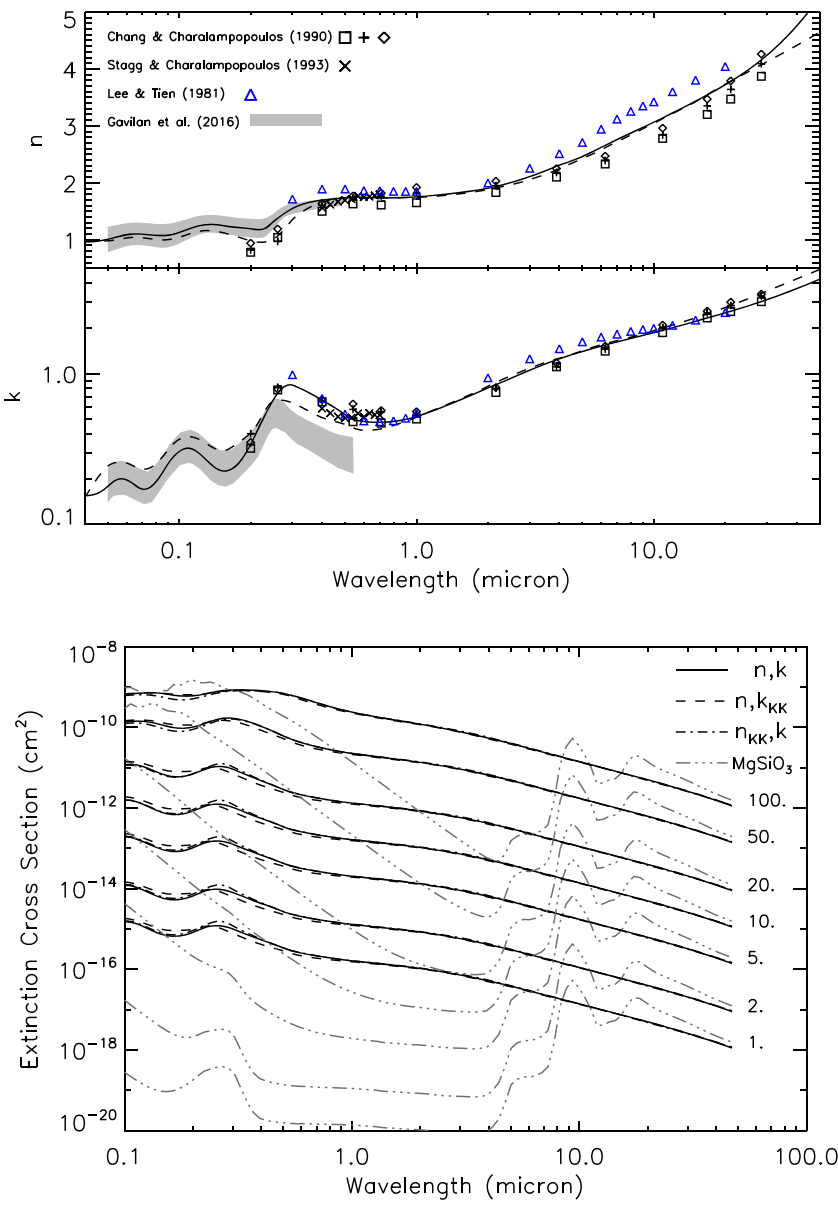

Figure 9. Top: compilation of refractive index measurements of soot particles from different laboratory studies (symbols). The solid lines represent the average spectra for the real and imaginary parts, and the dashed lines the calculated components based on the Krammers-Kroning dispersion formulae. Bottom: extinction cross-section of soot-composition particles at different radii (in $\mathrm{nm}$ ). Different curves correspond to different evaluations of the refractive index (see the text). The gray lines represent the equivalent cross-section assuming silicate composition $\left(\mathrm{MgSiO}_{3}\right)$.

reduced relative to our previous calculations of a clear atmosphere, since a fraction of the available photons at each atmospheric location is absorbed (or partially scattered) by the

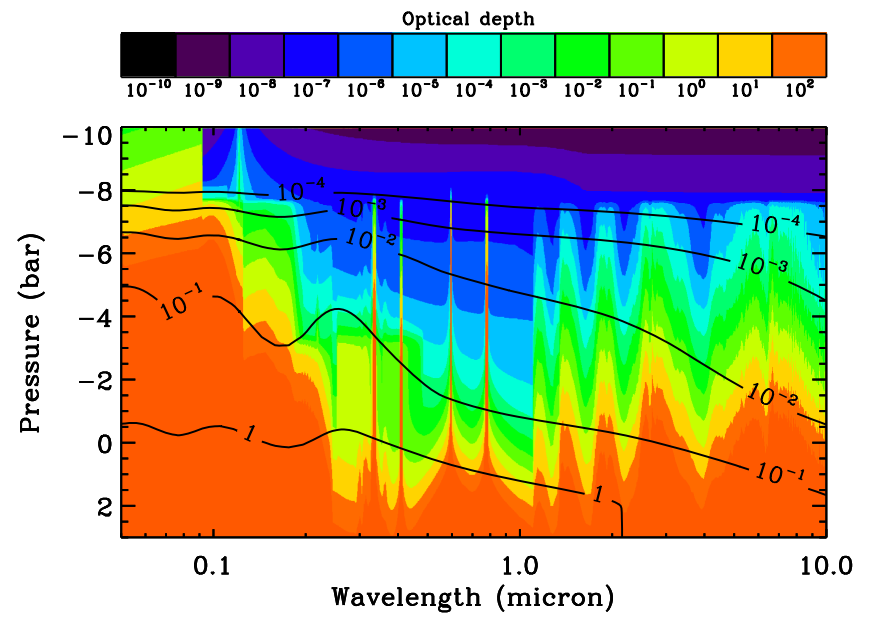

Figure 10. Wavelength-dependent atmospheric opacity at different locations in the atmosphere of HD $189733 \mathrm{~b}$ for the M profile and under nominal eddy conditions. The background colored contours represent the contribution of gaseous components, while the foreground black line contours correspond to the aerosol extinction contribution.

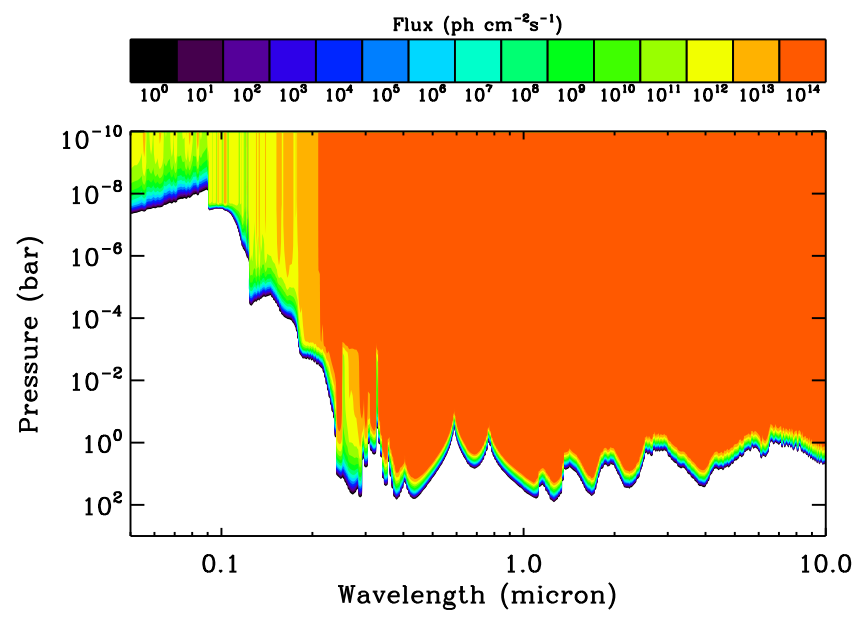

Figure 11. Contour plot of actinic fluxes vs. wavelength in the atmosphere of HD 189733 b for disk-average simulation (M profile).

aerosols. Thus, the abundances of main species such as $\mathrm{CH}_{4}$, $\mathrm{NH}_{3}$, and $\mathrm{H}_{2} \mathrm{~S}$ that have cross-sections in the wavelength range of aerosol influence are increased. Secondary products (e.g., $\mathrm{C}_{2} \mathrm{H}_{2}, \mathrm{~S}_{2}$ ) that have a photolysis cross-section that extend farther in the FUV are also directly affected by the aerosol opacity, but also indirectly by the changes the aerosols impose on the chemical abundances of intermediate species leading to their formation.

For the simulated aerosol opacity required to match the transit observations, the impact on the disk-average composition results is small and pertains mainly to the methane profile. However, the longer path lengths of the limb geometry increase the contribution of the aerosol opacity, and therefore the impact on the gaseous composition is larger for this case (Figure 12).

The simulated changes in the atmospheric composition from the inclusion of the aerosol opacity mainly concern the upper part of HD 189733 b's atmosphere. Therefore, they do not affect the interpretation of broadband observations from space telescopes, which are sensitive to pressures greater than 1-10 $\mu \mathrm{bar}$, depending on the thermal structure assumed (see Figure 7). However, for high-resolution ground-based observations, as, for 

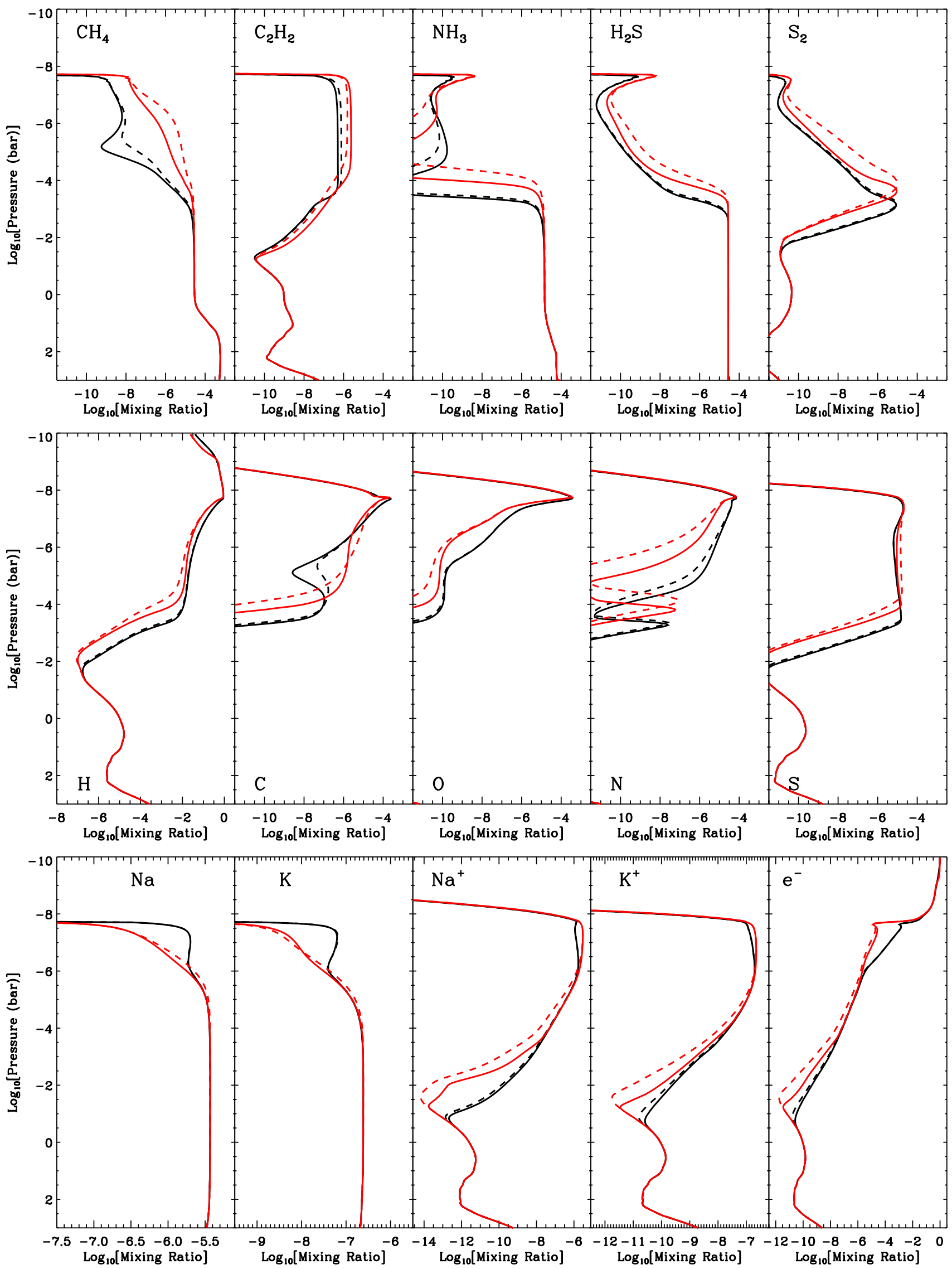

Figure 12. Mixing ratio profiles for different species in the atmosphere of HD $189733 \mathrm{~b}$ assuming the $\mathrm{M}$ profile. The black and red lines correspond to disk-average and limb-geometry conditions, while the dashed and solid curves represent the results with and without the impact of aerosols on the radiation field. 
example, the Na transit observations (Wyttenbach et al. 2015), the modifications of the $\mathrm{Na}$ profile imposed by the aerosols (Figure 12) could have an observable signature, given the sensitivity of the transit at the line cores to the $\mathrm{Na}$ abundance in the upper atmosphere. We will investigate this in more detail in a forthcoming study.

\subsection{Atmospheric Heating}

Aside from their impact on the photochemistry, aerosols can have a major influence on the atmospheric thermal structure, as indicated by their opacity at visible wavelengths, relative to the contribution of gases. In order to evaluate their impact, we calculated atmospheric heating rates from the major gaseous absorbers $\left(\mathrm{H}_{2} \mathrm{O}, \mathrm{CO}, \mathrm{CO}_{2}\right.$, and $\left.\mathrm{CH}_{4}\right)$, as well as heating from aerosol absorption (Figure 14). For the gaseous heating, we considered the absorption of stellar radiation from the molecular bands, taking into account the attenuation of the incoming radiation from all atmospheric components, including extinction at the $\mathrm{Na}$ and $\mathrm{K}$ resonant lines and Rayleigh scattering by atomic and molecular hydrogen.

For the aerosol heating rate, we cannot assume that all absorbed radiation is transferred to the atmosphere as heat because the aerosol population occupies a low-density part of the atmosphere. At these conditions, the particles are heated through absorption of stellar radiation but if collisions are not rapid enough to equilibrate their temperature with the background atmosphere, part of the absorbed energy will be emitted as IR radiation that will partially escape to space. Therefore, the atmospheric heating efficiency due to the aerosols will decrease with lower atmospheric density. To evaluate this parameter, we calculated the surface temperature of each particle in our size distribution at each atmospheric location by equilibrating the local heating and cooling rates. Heating is due to the absorption of the incoming stellar radiation, while cooling occurs due to thermal emission at the particle's surface temperature (considered to be a blackbody with an emission efficiency equal to the absorption efficiency at each wavelength) and energy transfer through collisions with the bulk atmospheric molecules. For the last term, we considered the thermal conductivity of a $\mathrm{H} / \mathrm{H}_{2}$ gas mixture, complete thermal accommodation $\left(\alpha_{\mathrm{T}}=1\right)$, and transition from the continuous to the free-molecular regime in our evaluation of the molecular collision rates with aerosol particles (see Lavvas et al. 2011a for more details). Our calculations indicate that the particle temperature starts to diverge from the atmospheric background temperature above 10 mbar for the largest particles resulting from our simulations, and thus the heating efficiency of the particles decreases at lower pressures (Figure 13). A small part of the emitted IR radiation may be reabsorbed by the atmosphere, thus our calculations provide a minimum estimate for the impact of the aerosols on the atmospheric heating rate.

The simulated energy deposition rates demonstrate that $\mathrm{H}_{2} \mathrm{O}$ provides the major heating in the lower atmosphere near the 1 bar level (Figure 14). However, at lower pressures (below $\sim 10 \mathrm{mbar}$ ), the photochemical aerosols dominate over all atmospheric heating contributions. This conclusion applies to both temperature profiles considered, although for the lowertemperature $\mathrm{M}$ profile, in which aerosols can survive to deeper pressures and reach higher particle sizes, their corresponding atmospheric heating is higher and dominates over the $\mathrm{H}_{2} \mathrm{O}$ contribution in the whole atmosphere. The relative contributions of the gaseous opacities also vary between the two atmospheric profiles considered. For example, heating by
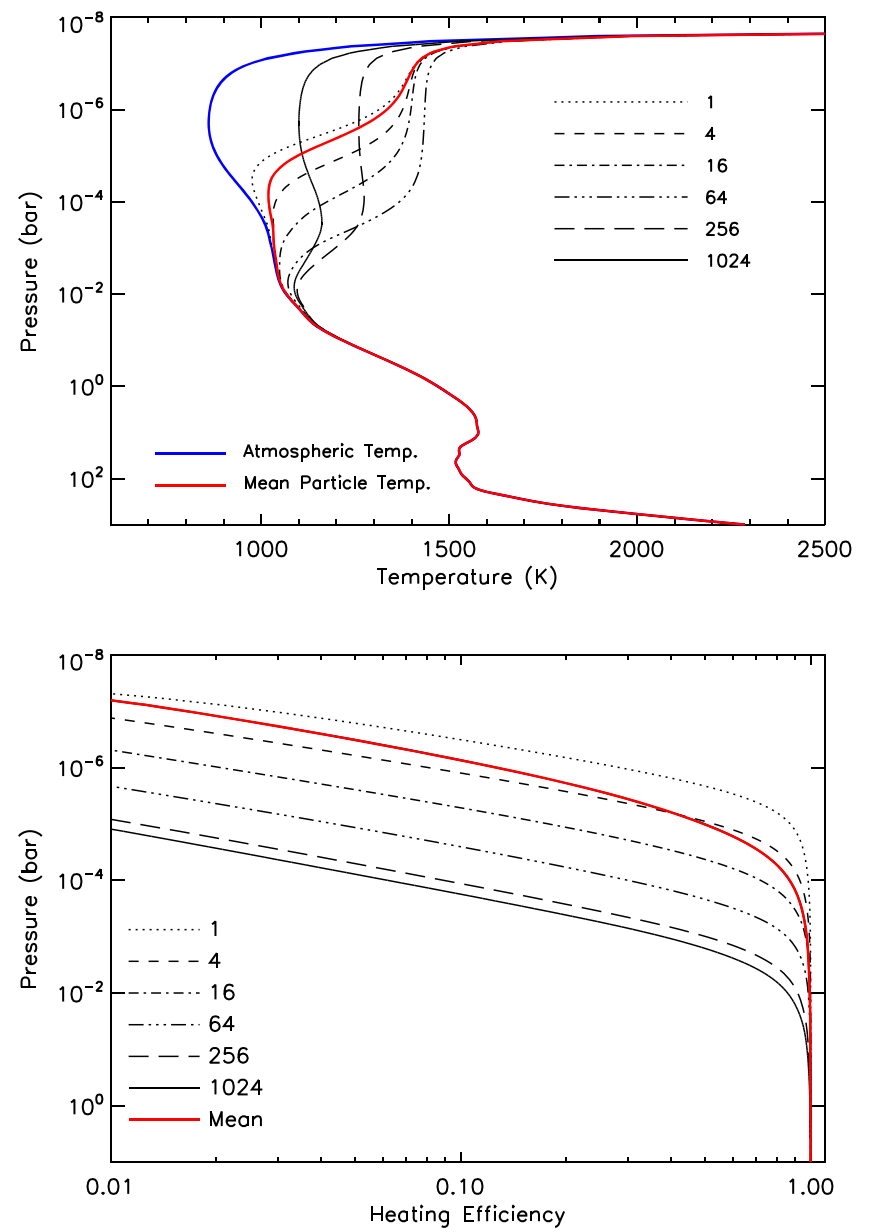

Figure 13. Top: particle temperatures (black lines) for different radii (in $\mathrm{nm}$ ) relative to the atmospheric background temperature (blue). The red line represents the average particle temperature based on the simulated size distribution. Bottom: particle heating efficiencies (black lines) at different locations in the atmosphere of HD $189733 \mathrm{~b}$ and the corresponding average profile (red line).

methane is second after water vapor for the $\mathrm{M}$ profile, but is practically insignificant for the $\mathrm{L}$ profile. This is a direct reflection of the different methane abundances allowed for each profile, as described above. Irrespective of the atmospheric structure assumed, we see that the presence of photochemical aerosols could significantly affect the atmospheric thermal structure and therefore contribute in merging the theoretical and retrieved temperature profiles. Thus, their impact should be considered in future studies of EGP thermal structure.

\section{External Sources}

So far, we have considered processes within the atmosphere that could affect the formation of photochemical aerosols. However, the contribution of external sources such as meteoroids should be evaluated. Meteoroids in the solar system have a composition reflecting their formation region, which mainly includes a metallic core dominated by iron and silicate minerals covered by ices (Jarosewich 1990). At the orbital distances of hot Jupiters $(\lesssim 0.1 \mathrm{au})$, the surface temperature of the incoming particles will be high and the icy coating of the meteoroids will evaporate. Thus, if we consider only metallic meteoroids and assume that their composition is dominated by silicates, we can evaluate their 

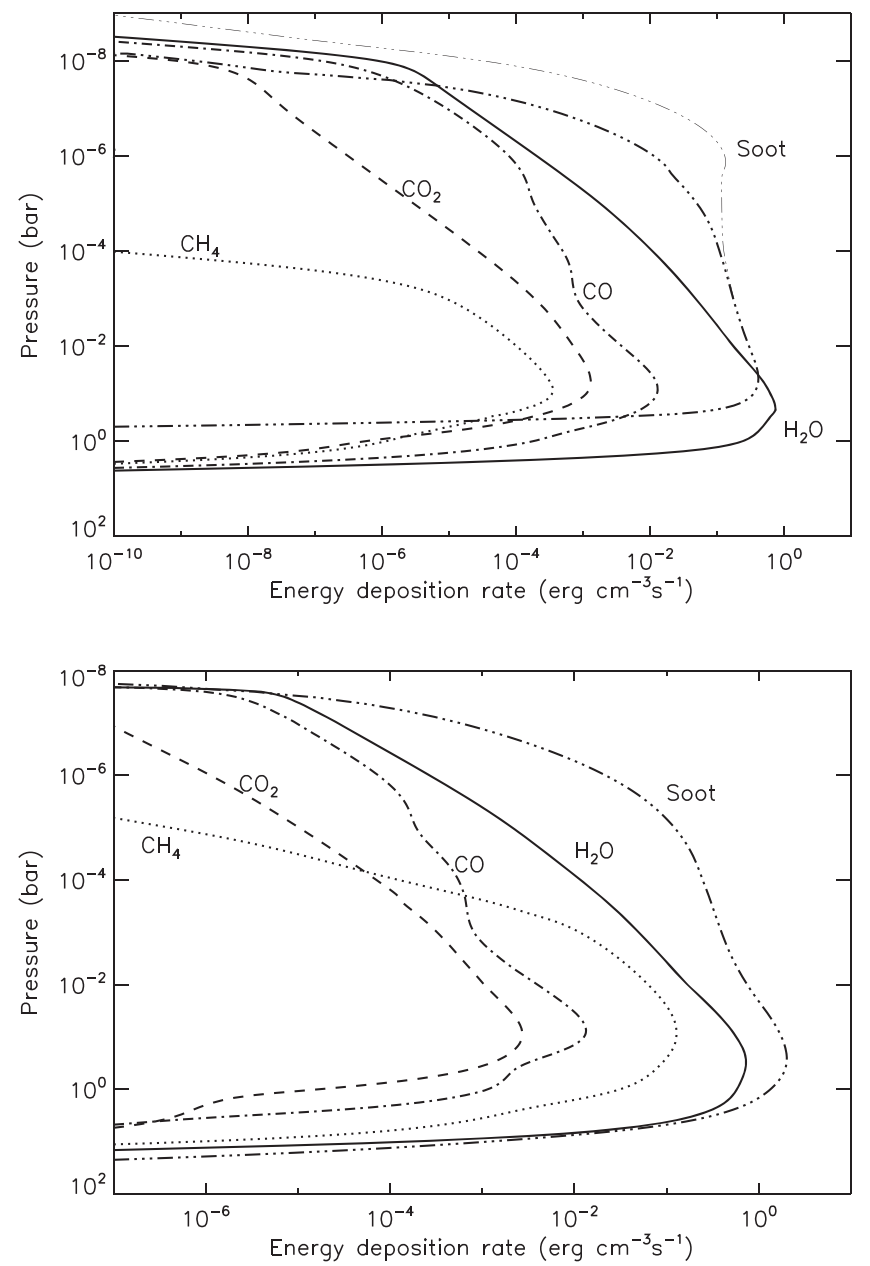

Figure 14. Energy deposition rates from the absorption of visible and near-IR radiation by the major gaseous components and the aerosols. The top panel presents the results for the $\mathrm{L}$ profile and the bottom panel for the M profile (see the text). The thick and thin lines for the aerosol contribution in the $\mathrm{L}$ case represent the resulting atmospheric heating with and without the inclusion of the particle heating efficiency.

surface temperature at different orbital distances by equilibrating the stellar energy they absorb with the energy they emit as thermal radiation and the latent heat lost during evaporation, if they reach a high enough temperature for this process to be important (Moses 1992). Assuming a spherical shape, we find that for radii smaller than $40 \mu \mathrm{m}$, meteoroids reach the orbit of HD 189733 b without suffering significant evaporation, while the corresponding size for HD $209458 \mathrm{~b}$ is $20 \mu \mathrm{m}$ due to the stronger flux of its star (Figure 15). Larger-size meteoroids evaporate faster as they absorb photons more efficiently.

This approach assumes steady-state conditions, which is a valid approximation for small particles as their characteristic time to reach equilibrium is small. Large-size meteoroids are rare events although their frequency will depend on the age of the stellar system. For HD 209458 b and HD 189733 b, with ages of $6.5 \pm 2.7 \mathrm{Gyr}$ and $4.3 \pm 2.8 \mathrm{Gyr}$, respectively (Boyajian et al. 2014), the meteoroid size distribution would probably be dominated by small-size particles $(\leqslant 1 \mathrm{~mm})$. Thus, it is likely that at the orbit of EGPs a significant fraction of meteoroids will ablate, providing a local source of silicate components such as $\mathrm{Si}$ and $\mathrm{Mg}$, while gravitational focusing could allow for a local increase of this silicate cloud in the vicinity of the planet. However, the importance of this effect in
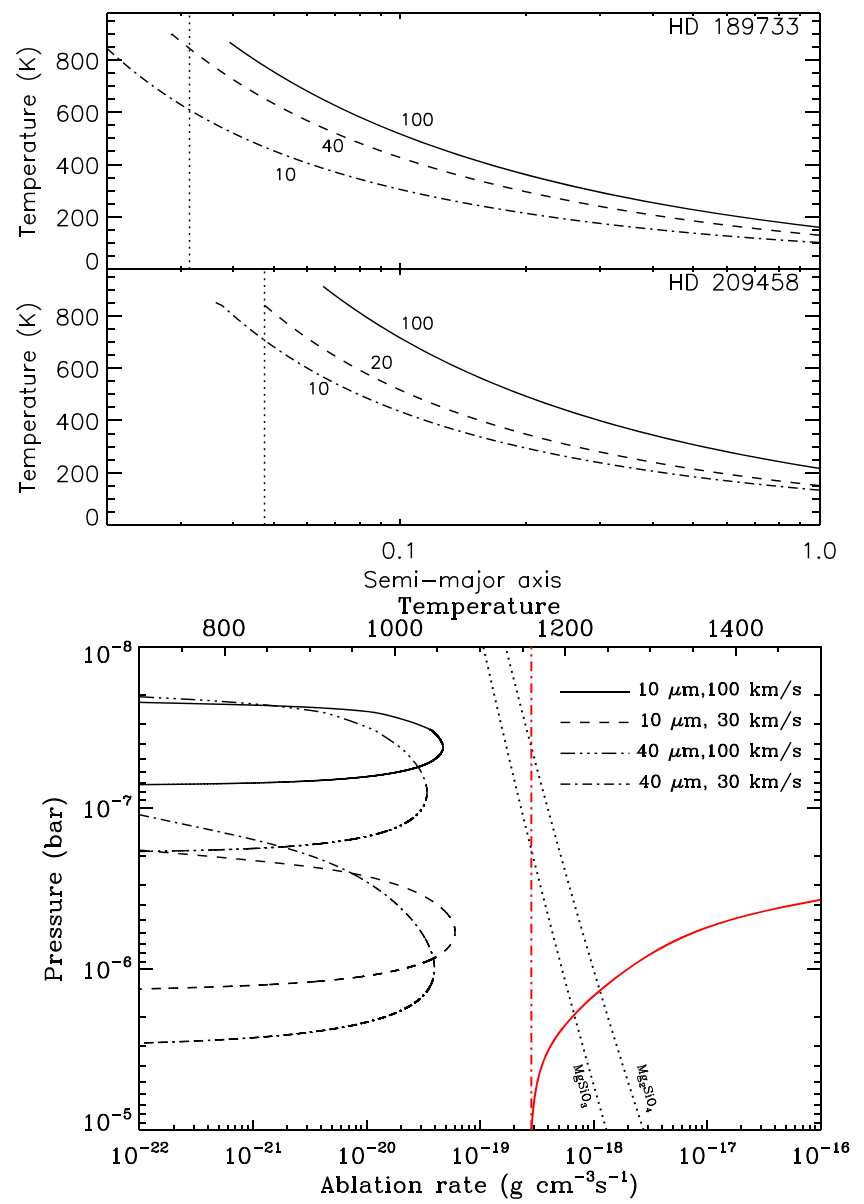

Figure 15. Top: variation of meteoroid surface temperature and radius at different orbital distances around HD 189733 and HD 209458. Each curve represents a different initial radius as designated (in $\mu \mathrm{m}$ ), and meteoroids are considered to have a silicate composition. Bottom: meteoroid ablation rate in the atmosphere of HD 189733 b for different meteoroid radii and impact velocities (black curves). The incoming meteoroid mass flux is assumed to be $10^{-12} \mathrm{~g} \mathrm{~cm}^{-2} \mathrm{~s}^{-1}$. The red lines represent the thermal structure for the $\mathrm{L}$ and LK profiles in the region of the atmosphere where meteoroids ablate. Dotted lines represent the condensation curves for silicate (see Figure 3).

the interpretation of the observed transit signatures of such elements (Linsky et al. 2010; Vidal-Madjar et al. 2013) depends on the meteoroid mass flux for which there is limited information.

We can further evaluate the impact such meteoroids would have once they enter the atmosphere of HD 189733 b. For this evaluation, we need to know the velocity of the meteoroids relative to the planet and the incidence angle at which they enter the atmosphere. An upper limit for the orbital velocity of the meteoroids would be their escape velocity from the star's gravity at the orbit of the planet, which for HD $189733 \mathrm{~b}$ is $\sim 200 \mathrm{~km} \mathrm{~s}^{-1}$. The actual relative velocity could range between zero and this limit, and in our calculations we estimated relative velocities of 30 and $100 \mathrm{~km} \mathrm{~s}^{-1}$. For the incidence angle, we assume that the meteoroids enter at $45^{\circ}$. In reality, meteoroids will have a distribution over size, relative velocity, and incidence angle, but for the simple test we wish to perform here, singular values will suffice. Moreover, the current understanding for the meteoroid distribution properties in extrasolar systems is rather limited, and using estimates based on solar system parameters can be highly uncertain given that 
such distributions will depend on the whole planetary system distribution and its age.

We calculate the ablation rate of different meteoroids by evaluating the variation of the temperature, velocity, and size once they enter the atmosphere, following the approach outlined in Moses (1992). Meteoroid temperature quickly increases due to collisions with the atmospheric molecules, and once they reach the evaporation temperature, they start to lose mass. Our results demonstrate that at high relative velocity impacts of the order of $100 \mathrm{~km} \mathrm{~s}^{-1}$, the meteoroid particle temperature increases faster than at the lower relative velocity $\left(30 \mathrm{~km} \mathrm{~s}^{-1}\right)$. As a result, ablation for the former case occurs at pressures lower that $0.1 \mu \mathrm{bar}$, while for the latter, particles evaporate slower and they deposit their mass near the $1 \mu \mathrm{bar}$ pressure region (Figure 15). For the same relative velocity, larger-size meteoroids provide broader ablation profiles because their temperature increases faster due to their larger cross-section.

The fate of the ablated material will strongly depend on the background atmospheric conditions and the abundance of the incoming meteoroids. If the temperature is low enough to allow the re-condensation of the ablated mass, silicate particles could form and affect the local extinction of the atmosphere. Apart from re-condensation, if the particle's velocity is thermalized before complete ablation (as we find for smaller-size meteoroids of the order of $1 \mu \mathrm{m}$ ), these could directly contribute to the atmospheric opacity. Therefore, there are two critical parameters that control the importance of external sources: the incoming flux of meteoroids and the thermal structure of the upper atmosphere. Unfortunately, both parameters are not accurately constrained currently.

However, the strong absorption near $10 \mu \mathrm{m}$ due to the $\mathrm{Si}-\mathrm{O}$ bond in silicate materials makes the absorption cross-section of such particles as strong as the cross-section at visible wavelengths (see Figure 9). Thus, the resulting transit depth from such particles near $10 \mu \mathrm{m}$ would be similar to the transit depth in the visible. Such a characteristic, however, is not supported by observations, thus limiting the contribution of silicates in their explanation. Other metals could exist in the ablated material, such as $\mathrm{Ti}, \mathrm{Al}$, and $\mathrm{Fe}$, which also form bonds with oxygen and result in a strong absorption feature near $10 \mu \mathrm{m}$ comparable with the absorption in the visible. Therefore, we can conclude that meteoroid ablation is probably not responsible for the observed transit depth of HD 189733 b. It could be important though for other exoplanets depending on the influx and atmospheric conditions. Moreover, even if it is not evident in the observations, meteoroid ablation could have other implications for the atmospheric properties, such as local ionization and cloud formation. As molecules collide with high-velocity meteoroids, they can be ionized, while the residual or recondensed meteoroid mass could act as a nucleation site for the formation of clouds deeper in the atmosphere.

\section{Discussion}

Our calculations demonstrate that soot-composition aerosols can match the primary transit observations of HD 189733 b's upper atmosphere, while the chemical composition results suggest that the photolysis mass fluxes from the major $\mathrm{CH}_{4}$ products are large enough to support the formation of soot particles. However, are the intermediate steps accessible at the conditions found in EGPs? Studies of soot formation in combustion experiments demonstrate that both aromatic and aliphatic structures are involved in the formation of nascent soot particles, while multiple mechanisms for the formation of such structures at high-temperature conditions have been suggested (Wang 2011). Recent laboratory investigations demonstrate that $\mathrm{CO}$ can also increase the efficiency of aerosol production in experiments of various energy inputs (Horst \& Tolbert 2014), although the mechanisms behind this interaction needs to be further investigated. Thus, the high abundance of $\mathrm{CO}$ in EGPs could further contribute to photochemical aerosol formation.

A limiting factor for the aerosol formation could be the oxidation of the atmosphere that will be controlled dominantly by the $\mathrm{OH}$ radicals produced in the dissociation of $\mathrm{H}_{2} \mathrm{O}$. Studies at combustion conditions demonstrate that the addition of water vapor reduces the soot formation efficiency because the reaction of $\mathrm{OH}$ with hydrocarbons limits their growth. A comparison of the $\mathrm{OH}$ radical abundance to that of the $\mathrm{C} / \mathrm{N}$ composition radical species responsible for the growth of hydrocarbons (an approach similar to Morley et al. 2013 for the evaluation of the oxidation impact) demonstrates that the latter can dominate over the former depending on the thermal structure and atmospheric pressure (Figure 16). This preliminary test indicates that soot formation could be possible in hot exoplanets, but a more thorough investigation considering the reactivity of each species and the impact of the radiation field is required. Nevertheless, our conclusion is in agreement with previous studies for the chemical composition of EGPs, which demonstrate that reduced carbon chemistry is possible for $\mathrm{C} / \mathrm{O}<1$ at high $K_{\mathrm{ZZ}}$ values (Moses et al. 2013; Zahnle \& Marley 2014). Moreover, studies of aerosol formation in Titan's upper atmosphere demonstrate that ion-neutral processes have a dominant role in the formation of the nascent aerosols found there, and the role of similar mechanisms on exoplanet ionospheres needs to be evaluated. Such a study goes beyond the goals of the current preliminary investigation and will be addressed in a future work.

Although our results demonstrate that a photochemical aerosol distribution of soot composition can reproduce the primary eclipse observations for HD $189733 \mathrm{~b}$, this result does not contradict the anticipated clouds of silicate composition in the deeper atmosphere. The latter are still likely to form in the deeper atmosphere, but will not necessarily be responsible for the heterogeneous opacity observed in the primary eclipse observations. However, the photochemical aerosols formed in the upper atmosphere of HD 189733 b could have an important role in the resulting properties of silicate clouds in the lower atmosphere as they can act as nucleation sites. Current models for the formation of silicate composition clouds consider condensed $\mathrm{TiO}_{2}$ as nucleation centers (Lee et al. 2017), the latter formed through the homogeneous nucleation of the gas phase. Heterogeneous nucleation is usually orders of magnitude faster than homogeneous nucleation; therefore, it could significantly modify the resulting cloud particle properties. Moreover, homogeneous nucleation depends strongly on the thermal structure, and it would be eliminated if the atmosphere includes the thermospheric temperature increase suggested by the atmospheric escape models. Thus, the impact of heterogeneous nucleation on silicate condensation could also modify the vertical extent of the formed clouds. Therefore, the impact of photochemical aerosols as well as that of possible meteoroid 


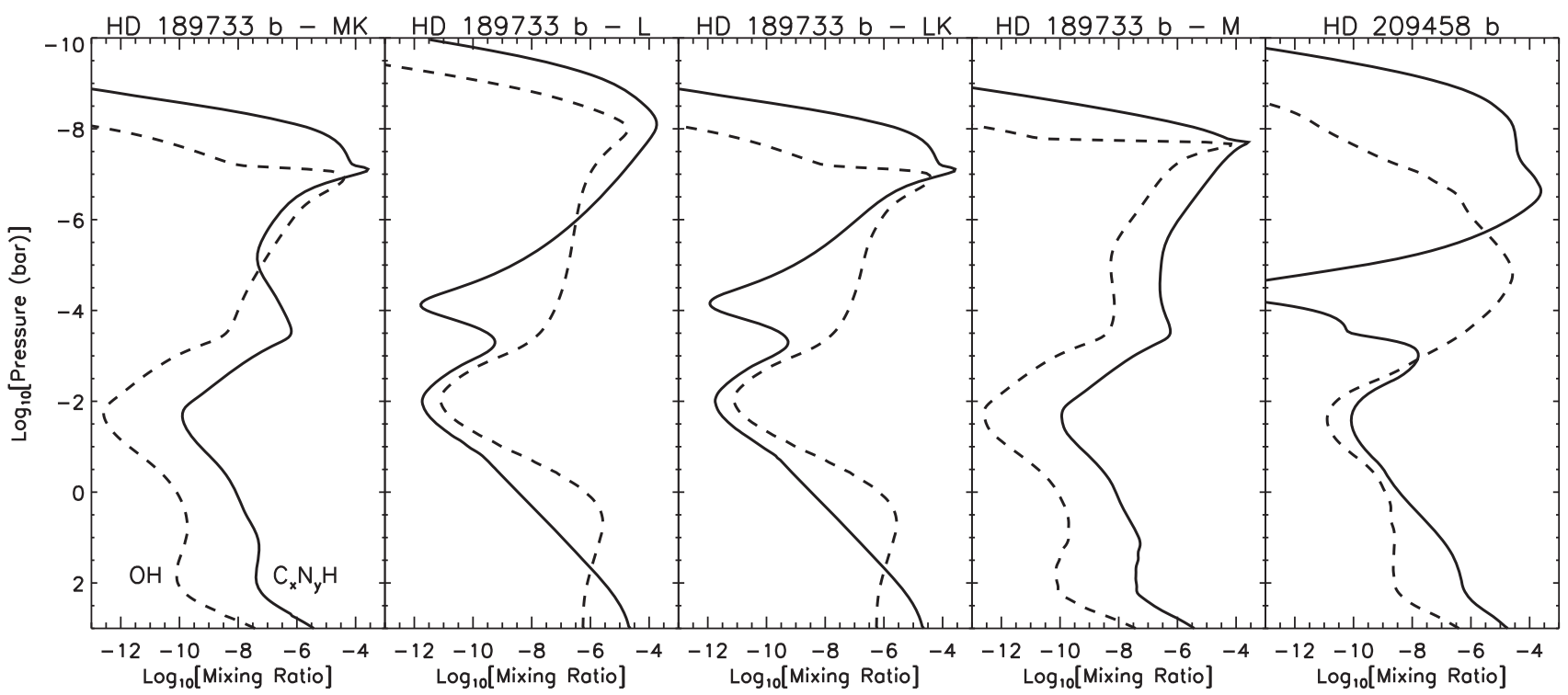

Figure 16. Comparison of $\mathrm{OH}$ (dashed) and carbon-based radical (solid) mixing ratios for the different $p-T$ profiles assumed in the atmospheres of $\mathrm{HD} 189733 \mathrm{~b}$ and HD 209458 b.

ablation products should be considered in future cloud nucleation simulations.

Current observational constraints as well as the accuracy of theoretical studies do not allow the thermal structure and turbulent mixing in the atmospheres of EGPs to be retrieved. Thus, among the different scenarios we investigated, we can find solutions that provide fits to the observations. However, we note that the best fits in terms of the reduced $\chi^{2}$ (Table 2) suggest that the nominal values of the eddy mixing profile provide aerosol distributions that fit the observations better. Similarly, the hotter thermal structure derived from the inversion of the secondary eclipse observations (L profile) also improve the fit to the observations relative to the GCM temperature profile (M profile), although the former is based on an ad hoc extension of the derived thermal structure at pressures away from the range probed in the secondary eclipse. These characteristics have further ramifications for our understanding of the shape of the particles, their charging, as well as their interaction with the atmosphere of HD $189733 \mathrm{~b}$.

With regard to the particle shape, we found earlier that the nominal eddy profile results in smaller aerosol particle sizes with average particle radii ranging between 1.5 and $2 \mathrm{~nm}$ for the pressure range probed in the UV part of the spectrum (see Table 2). As discussed above, this weak growth of aerosol particles is driven by the strong atmospheric mixing that efficiently transports the particles to deeper pressures, thereby limiting their collisions. At these small radii, particles are near their embryonic size, as this is defined by the chemical mechanisms leading to their formation. Thus, their size will still be affected by the gas-phase chemistry as anticipated in both Titan's upper atmosphere (Lavvas et al. 2011b) and observed in soot formation laboratory experiments (Wang 2011). Thus, the effectively weak growth through coagulation for the nominal eddy profile and the small particle size indicate that the photochemical aerosols in the upper atmosphere of HD $189733 \mathrm{~b}$ are most likely spherical rather than aggregates. However, in the lower atmosphere where collisions are more frequent particles could form aggregates. The same applies for cloud particles if the mass growth rate through their collisions dominates over their growth through condensation.

So far, we have assumed that the particle growth is not affected by charge effects. This assumption is justified for the small particle sizes that we calculate for the nominal $K_{\mathrm{ZZ}}$ case because particles of a few nanometer radius will be able to acquire a limited number of charges (Lavvas et al. 2013). On the other hand, the small size of the particles could potentially allow them to be drifted by electric fields in the atmosphere. A complete investigation of the problem requires a coupled investigation of aerosols, clouds, and atmospheric background, which is beyond the goal of our current study.

The higher temperature of the $\mathrm{L}$ profile makes the atmospheric scale height larger, and therefore makes the slant opacity larger relative to the M profile case. As a result, the former profile provides a better fit to the observations than the latter. The discrepancy between the theoretical (M) and retrieved (L) temperature structures suggests that a heating mechanism is required to bring the two approaches into better agreement. Our calculations demonstrate that heating by photoabsorption from soots is significant and could lead to hotter temperatures than predicted by current models. We further note that irrespective of its composition, the heterogeneous opacity indicated by the primary transit observations is likely to affect both the local thermal structure and the radiation field, and thus the atmospheric photochemistry.

Current escape calculations usually set their high-pressure boundary near the $1 \mu$ bar pressure level or at lower pressures, and evaluate the thermal structure of the upper atmosphere considering mainly $\mathrm{H}_{2}$ and other atomic contributions, but not the impact of $\mathrm{HCN}, \mathrm{C}_{2} \mathrm{H}_{2}$, and other strong molecular coolants. However, our model results demonstrate that the abundances of such molecules are important in the middle and upper atmospheres and can potentially affect the local temperature and the associated escape rates. Our simulated composition results do not consider the impact of the bulk escape on the mixing ratio profiles, which could affect the distribution of species in the upper atmosphere. Therefore, the abundances of some species could be even higher than predicted, if the temperature conditions allow their survival. However, since our 
goal here is only to evaluate the production rate of photochemical aerosols that mainly depends on the column photolysis rate of different molecules, we consider that the actual shape of the species profiles in the upper atmosphere will not significantly modify our estimates. Nevertheless, the impact of molecular abundances on the thermal structure needs to be evaluated in future investigations, also taking into consideration the non-LTE character of the upper atmosphere. This aspect is not considered in the current thermal structure models, and its importance will be enhanced if molecular structures survive to significant abundances.

We saw that soot particles vaporize at significantly higher temperatures relative to other compounds (e.g., of sulfur composition), which makes them a suitable candidate for the high-temperature conditions found in HD 189733 b's atmosphere. The impact of particle decomposition affects a part of the atmosphere that is not probed in the primary transit observations, depending on the reference pressure level of the atmosphere. However, particle decomposition can have significant ramifications for the interaction of these particles with the background atmosphere. One example is the role of aerosols in cloud formation discussed above; another example is the heterogeneous chemical processes on their surface, which could have an impact on the gas-phase composition. These are aspects that should be investigated in future studies and their impact evaluated against secondary eclipse observations.

\section{Conclusions}

We presented a detailed study of the possible properties of photochemical aerosols in the atmospheres of EGPs taking into account internal and external sources that could affect their production. Our results demonstrate that:

1. The thermal structure in the middle atmosphere significantly affects the chemical composition and survival of molecular structures that could lead to the formation of photochemical aerosols. Moreover, the temperature profile controls the stability of possible aerosols against thermal decomposition.

2. Among the possible candidates we considered, soot has the highest resistance to high temperatures and is a possible product in hot Jupiters based on the aerosol mass production rates estimated from the photochemical model results for HD $189733 \mathrm{~b}$. Hotter temperatures in the upper atmosphere of HD 209458 b can explain the apparent lack of photochemical aerosols in its transmission spectrum.

3. Aerosol mass fluxes of the order of $10^{-12} \mathrm{~g} \mathrm{~cm}^{-2} \mathrm{~s}^{-1}$ are required to match the primary transit observations of HD $189733 \mathrm{~b}$, assuming the nominal eddy mixing profile, while for lower mixing efficiency, the required mass flux is reduced as particles grow to larger sizes that result in larger extinction cross-sections.

4. The average particle size for photochemical aerosols depends strongly on the efficiency of the atmospheric mixing assumed. For the nominal eddy profiles assumed in the literature according to GCMs, transport is more efficient than collisions, and the resulting particle size is small, between 1 and $2 \mathrm{~nm}$, in the region of the atmosphere probed by the transit observations. For these conditions, particles will most likely be spherical, and their charging will not significantly affect their size distribution. If however, atmospheric mixing is weaker, particles can grow to large sizes reaching up to $100 \mathrm{~nm}$ for the highest mass fluxes considered. In this case, aggregation is possible and charge effects need to be evaluated.

5. The soot aerosols that match the primary transit observations would provide significant opacity over the stellar spectrum and contribute significantly to atmospheric heating. However, the overall contribution of such particles to the thermal structure needs to be evaluated through detailed models. Moreover, soot aerosols also affect the transfer of radiation at short wavelengths and thus the atmospheric photochemistry, leading to changes in the gaseous abundances. This effect will be more pronounced at the terminators where the aerosol slant optical depth is higher.

6. Meteoroid ablation provides a mass input in the upper atmosphere of EGPs, but the spectral signature of the heterogeneous compounds, primarily silicates, that could form is not consistent with the observations of HD 189733 b. Thus, the in situ atmospheric composition should control aerosol formation.

We thank R.V. Yelle for his comments on the manuscript. P.L. acknowledges financial support from the Programme National de Planétologie (PNP-INSU) under project AMG and from the Projet International de Coopération Scientifique (PICS/CNRS) under project TAC.

\section{References}

Agol, E., Cowan, N. B., Knutson, H. A., et al. 2010, ApJ, 721, 1861

Ben-Jaffel, L., Leers, V., \& Sandel, B. R. 1995, Sci, 269, 951

Boyajian, T., von Braun, K., Feiden, G. A., et al. 2014, MNRAS, 447, 846

Brown, T. M. 2001, ApJ, 553, 1006

Cameron, A. G. W. 1985, Icar, 64, 285

Chang, H., \& Charalampopoulos, T. T. 1990, RSPSA, 430, 577

Chokshi, A., \& Tielens, A. 1993, ApJ, 403, 806

Désert, J.-M., Lecavelier des Etangs, A., Hébrard, G., et al. 2009, ApJ, 699,478

Désert, J.-M., Vidal-Madjar, A., Lecavelier Des Etangs, A., et al. 2008, A\&A, 492, 585

Diamond-Lowe, H., Stevenson, K. B., Bean, J. L., Line, M. R., \& Fortney, J. J. 2014, ApJ, 796, 66

Dominik, C., \& Tielens, A. G. G. M. 1997, ApJ, 480, 647

Draine, B. T. 2004, in Carnegie Observatories Centennial Symp., Origin and Evolution of the Elements, ed. A. McWilliam \& M. Rauch (Cambridge: Cambridge Univ. Press), 317

Fortney, J. J., Sudarsky, D., Hubeny, I., et al. 2003, ApJ, 589, 615

Frenklach, M. 2002, PCCP, 4, 2028

García Muñoz, A. 2007, P\&SS, 55, 1414

Gavilan, L., Alata, I., Le, K. C., et al. 2016, A\&A, 586, A106

Gibson, N. P., Pont, F., \& Aigrain, S. 2011, MNRAS, 411, 2199

Goldfarb, J. L., \& Suuberg, E. M. 2008, Journal of Chemical \& Engineering Data, 53, 670

Grillmair, C. J., Burrows, A., Charbonneau, D., et al. 2008, Natur, 456, 767

Harris, S. J., \& Kennedy, I. M. 1988, CST, 59, 443

Helling, C., Jardine, M., Diver, D., \& Witte, S. 2013, P\&SS, 77, 152

Helling, C., \& Woitke, P. 2006, A\&A, 455, 325

Hora, J. L., Carey, S., Surace, J., et al. 2008, PASP, 120, 1233

Horst, S. M., \& Tolbert, M. A. 2014, ApJ, 781, 53

Hubeny, I., Burrows, A., \& Sudarsky, D. 2003, ApJ, 594, 1011

Jarosewich, E. 1990, Metic, 25, 323

Johnson, K. L., Kendall, K., \& Roberts, A. D. 1971, RSPSA, 374, 301

Koskinen, T., Harris, M., Yelle, R., \& Lavvas, P. 2013, Icar, 226, 1678

Koskinen, T. T., Aylward, A. D., \& Miller, S. 2007, Natur, 450, 845

Koskinen, T. T., Moses, J. I., West, R. A., Guerlet, S., \& Jouchoux, A. 2016, GeoRL, 43, 7895

Lavvas, P., Griffith, C. A., \& Yelle, R. V. 2011a, Icar, 215, 732

Lavvas, P., Koskinen, T., \& Yelle, R. V. 2014, ApJ, 796, 15 
Lavvas, P., Sander, M., Kraft, M., \& Imanaka, H. 2011b, ApJ, 728, 80

Lavvas, P., Yelle, R. V., \& Griffith, C. A. 2010, Icar, 210, 832

Lavvas, P., Yelle, R. V., Koskinen, T., et al. 2013, PNAS, 110, 2729

Lavvas, P. P., Coustenis, A., \& Vardavas, I. M. 2008, P\&SS, 56, 27

Lecavelier Des Etangs, A., Pont, F., Vidal-Madjar, A., \& Sing, D. 2008a A\&A, 481, L83

Lecavelier Des Etangs, A., Vidal-Madjar, A., Désert, J.-M., \& Sing, D. 2008b, A\&A, 485, 865

Lee, G., Helling, C., Dobbs-Dixon, I., \& Juncher, D. 2015, A\&A, 580, A12

Lee, G., Wood, K., Dobbs-Dixon, I., Rice, A., \& Helling, C. 2017, A\&A, 601, A22

Lee, S. C., \& Tien, C. L. 1981, Symposium (International) on Combustion, 18,1159

Lilov, S. K. 1993, MSEnB, 21, 65

Line, M. R., Knutson, H., Wolf, A. S., \& Yung, Y. L. 2014, ApJ, 783, 70

Line, M. R., Liang, M. C., \& Yung, Y. L. 2010, ApJ, 717, 496

Line, M. R., \& Parmentier, V. 2016, ApJ, 820, 78

Line, M. R., Stevenson, K. B., Bean, J., et al. 2016, AJ, 152, 203

Linsky, J. L., Yang, H., France, K., et al. 2010, ApJ, 717, 1291

Lyons, J. R. 2008, Journal of Sulfur Chemistry, 29, 269

Marley, M. S., Ackerman, A. S., Cuzzi, J. N., \& Kitzmann, D. 2013, Comparative Climatology of Terrestrial Planets, chapter Clouds and Hazes in Exoplanet Atmospheres (Tucson, AZ: Univ. Arizona Press), 367

Marley, M. S., Gelino, C., Stephens, D., Lunine, J. I., \& Freedman, R. 1999, ApJ, 513, 879

Marley, M. S., \& McKay, C. P. 1999, Icar, 138, 268

Michelsen, H. A., Liu, F., Kock, B. F., et al. 2007, ApPhB, 87, 503

Mitchell, P., \& Frenklach, M. 2003, PhRvE, 67, 061407

Moreno, F. 1996, Icar, 124, 632

Morgan, N., Kraft, M., Balthasar, M., et al. 2007, Proceedings Of The Combustion Institute, 31, 693

Morley, C. V., Fortney, J. J., Kempton, E. M. R., et al. 2013, ApJ, 775, 33

Moses, J. I., Madhusudhan, N., Visscher, C., \& Freedman, R. S. 2013, ApJ, 763,25

Moses, J. I., Visscher, C., Fortney, J. J., et al. 2011, ApJ, 737, 15

Moses, J. I. 1992, Icar, 99, 368

Oja, V., \& Suuberg, E. M. 1998, Journal of Chemical \& Engineering Data, 43, 486

Parmentier, V., Showman, A. P., \& Lian, Y. 2013, A\&A, 558, A91

Pinhas, A., \& Madhusudhan, N. 2017, MNRAS, 471, 4355
Pont, F., Knutson, H., Gilliland, R. L., Moutou, C., \& Charbonneau, D. 2008, MNRAS, 385, 109

Pont, F., Sing, D. K., Gibson, N. P., et al. 2013, MNRAS, 432, 2917

Rey, M., Nikitin, A. V., Babikov, Y. L., \& Tyuterev, V. G. 2016, JMoSp, 327, 138

Rowe, J. F., Matthews, J. M., Seager, S., et al. 2006, ApJ, 646, 1241

Showman, A. P., Fortney, J. J., Lian, Y., et al. 2009, ApJ, 699, 564

Sing, D. K., Désert, J.-M., Lecavelier Des Etangs, A., et al. 2009, A\&A, 505,891

Sing, D. K., Fortney, J. J., Nikolov, N., et al. 2016, Natur, 529, 59

Sing, D. K., Pont, F., Aigrain, S., et al. 2011, MNRAS, 416, 1443

Sudarsky, D., Burrows, A., \& Pinto, P. 2000, ApJ, 538, 885

Swain, M. R., Vasisht, G., \& Tinetti, G. 2008, Natur, 452, 329

Swain, M. R., Vasisht, G., Tinetti, G., et al. 2009, ApJL, 690, L114

Tennyson, J., Yurchenko, S. N., Al-Refaie, A. F., et al. 2016, JMoSp, 327, 73

Vardavas, I. M., \& Taylor, F. W. 2007, Radiation and Climate (Oxford: Oxford Univ. Press)

Vidal-Madjar, A., Huitson, C. M., Bourrier, V., et al. 2013, A\&A, 560, A54

Visscher, C., Lodders, K., \& Fegley, B. J. 2010, ApJ, 716, 1060

Wang, H. 2011, Proceedings Of The Combustion Institute, 33, 41

West, R. A., Baines, K. H., Friedson, A. J., et al. 2007, in Jupiter, Jovian clouds and haze, ed. F. Bagenal, T. E. Dowling, \& W. B. McKinnon (Cambridge: Cambridge Univ. Press), 79

West, R. A., Baines, K. H., Karkoschka, E., \& Sánchez-Lavega, A. 2009, in Saturn from Cassini-Huygens, Clouds and Aerosols in Saturn's Atmosphere, ed. M. K. Dougherty, L. W. Esposito, \& S. M. Krimigis (Berlin: Springer), 161

West, R. A., Lavvas, P., Anderson, C., \& Imanaka, H. 2013, in Titan, Titan's Haze, ed. I. Müller-Wodarg et al. (Cambridge: Cambridge Univ. Press), 285

Woitke, P., \& Helling, C. 2004, A\&A, 414, 335

Wong, A.-S., Lee, A. Y. T., Yung, Y. L., \& Ajello, J. M. 2000, ApJL, 534, L215

Wong, A.-S., Yung, Y. L., \& Friedson, A. J. 2003, GeoRL, 30, 1447

Wyttenbach, A., Ehrenreich, D., Lovis, C., Udry, S., \& Pepe, F. 2015, A\&A, 577, A62

Yelle, R. V. 2004, Icar, 170, 167

Zahnle, K., Marley, M. S., Freedman, R. S., Lodders, K., \& Fortney, J. J. 2009, ApJL, 701, L20

Zahnle, K., Marley, M. S., Morley, C. V., \& Moses, J. I. 2016, ApJ, 824, 137

Zahnle, K. J., \& Marley, M. S. 2014, ApJ, 797, 41 